\title{
A necessary and sufficient condition for finite speed of propagation in the theory of doubly nonlinear degenerate parabolic equations
}

\author{
B. H. Gilding \\ Faculty of Applied Mathematics, University of Twente, P.O. Box 217, \\ 7500 AE Enschede, The Netherlands \\ R. Kersner \\ Computer and Automation Research Institute, Hungarian Academy of \\ Sciences, P.O. Box 63, H-1518 Budapest, Hungary
}

(MS received 6 October 1994. Revised MS received 6 March 1995)

\begin{abstract}
A degenerate parabolic partial differential equation with a time derivative and first- and second-order derivatives with respect to one spatial variable is studied. The coefficients in the equation depend nonlinearly on both the unknown and the first spatial derivative of a function of the unknown. The equation is said to display finite speed of propagation if a non-negative weak solution which has bounded support with respect to the spatial variable at some initial time, also possesses this property at later times. A criterion on the coefficients in the equation which is both necessary and sufficient for the occurrence of this phenomenon is established. According to whether or not the criterion holds, weak travelling-wave solutions or weak travelling-wave strict subsolutions of the equation are constructed and used to prove the main theorem via a comparison principle. Applications to special cases are provided.
\end{abstract}

\section{Introduction}

This paper is devoted to the study of the property of finite speed of propagation of disturbances in solutions of the equations

$$
\frac{\partial u}{\partial t}=\frac{\partial}{\partial x}\left(a\left(u, \frac{\partial \varphi(u)}{\partial x}\right)\right)
$$

and

$$
\frac{\partial u}{\partial t}=\frac{\partial}{\partial x}\left(a\left(u, \frac{\partial \varphi(u)}{\partial x}\right)\right)+b\left(u, \frac{\partial \varphi(u)}{\partial x}\right) .
$$

The coefficients in equation (1.1) are assumed to satisfy the following hypothesis:

HYPOTHESIS 1.1. (i) $\varphi \in C\left(\overline{\mathbb{R}^{+}}\right) \cap W_{\text {loc }}^{1,1}\left(\mathbb{R}^{+}\right)$, $\varphi$ is strictly increasing on $\mathbb{R}^{+}$and $\varphi(0)=0$. (ii) $a \in C\left(\overline{\mathbb{R}^{+}} \times \mathbb{R}\right), a(u, \cdot)$ is strictly increasing on $\mathbb{R}$ for all $u \in \overline{\mathbb{R}^{+}}$and $a(0,0)=0$.

For equation (1.2), the coefficients will be assumed to satisfy slightly stronger conditions, namely the following:

Hypothesis 1.2. (i) $\varphi \in C\left(\overline{\mathbb{R}^{+}}\right) \cap C^{1}\left(\mathbb{R}^{+}\right), \varphi^{\prime}(u)>0$ for all $u \in \mathbb{R}^{+}$and $\varphi(0)=0$. 
(ii) $a \in C\left(\overline{\mathbb{R}^{+}} \times \mathbb{R}\right) \cap C^{0,1}\left(\overline{\mathbb{R}^{+}} \times \mathbb{R}^{+}\right) \cap C^{0,1}\left(\overline{\mathbb{R}^{+}} \times \mathbb{R}^{-}\right) \cap C^{1,0}\left(\mathbb{R}^{+} \times\{0\}\right), a_{v}(u, v)>0$ for all $(u, v) \in \overline{\mathbb{R}^{+}} \times \mathbb{R}^{+} \cup \overline{\mathbb{R}^{+}} \times \mathbb{R}^{-}$and $a(0,0)=0$.

(iii) $b \in C\left(\mathbb{R}^{+} \times \mathbb{R}\right) \cap C^{0,1}\left(\mathbb{R}^{+} \times \overline{\mathbb{R}^{+}}\right) \cap C^{0,1}\left(\mathbb{R}^{+} \times \overline{\mathbb{R}^{-}}\right), \quad b \varphi^{\prime} \in L^{1}\left(0, U ; L_{\mathrm{loc}}^{\infty}(\mathbb{R})\right)$ and $b_{v} \varphi^{\prime} \in L^{1}\left(0, U ; L_{\mathrm{loc}}^{\infty}(\mathbb{R})\right)$ for all $U \in \mathbb{R}^{+}, \quad \max \{0, b\} \in L^{\infty}\left(0, U ; L_{\mathrm{loc}}^{\infty}(\mathbb{R})\right)$ or $\max \{0,-b\} \in L^{\infty}\left(0, U ; L_{\mathrm{loc}}^{\infty}(\mathbb{R})\right)$ for all $U \in \mathbb{R}^{+}$, and $b(0,0)=0$.

For clarity in the above, $\mathbb{R}^{+}$denotes the set of positive real numbers and $\mathbb{R}^{-}$the set of negative real numbers, i.e. $\mathbb{R}^{+}=(0, \infty)$ and $\mathbb{R}^{-}=(-\infty, 0)$ in terms of intervals.

Typical examples of equation (1.1) are

$$
\frac{\partial u}{\partial t}=\frac{\partial}{\partial x}\left(\left|\frac{\partial u^{m}}{\partial x}\right|^{p-1} \frac{\partial u^{m}}{\partial x}\right)
$$

for some real parameters $m>0$ and $p>0$, and its generalisation

$$
\frac{\partial u}{\partial t}=\frac{\partial}{\partial x}\left(D\left(\frac{\partial \varphi(u)}{\partial x}\right) \frac{\partial \varphi(u)}{\partial x}\right),
$$

where $\varphi$ satisfies Hypothesis 1.1(i) and $D(v)$ is a continuous function which is positive for almost all $v$. These equations arise as models of gas flow through a porous medium in the turbulent regime [41-44]. Supposing that the flow is isothermal, the motion may be described by $\partial u / \partial t=\operatorname{div}(D \operatorname{grad} \varphi(u))$ where $u$ denotes the density of the gas, $t$ time, the coefficient $D$ is determined by experiment, and the function $\varphi$ is derived from the equation of state relating the pressure and density of the gas at constant temperature. For one-dimensional flow, this equation of motion takes the form (1.4). If, moreover, the gas is polytropic and the coefficient $D$ is given by the empirical relation $D(v)=D_{0}|v|^{p-1}$ for some positive constants $D_{0}$ and $p$, after a suitable rescaling of the variables, equation (1.4) reduces to (1.3) with a specific value of $m>1$. When $m \neq 1$ and $p \neq 1$, equation (1.3) is consequently sometimes known as the equation of non-Newtonian polytropic or turbulent filtration [40]. With $m=1$ and $p \neq 1$ it is also called the equation of non-Newtonian elastic filtration [40] or more commonly the heat equation with $p$-Laplacian. For $p=1$ and $m \neq 1$, the equation is widely known as the porous media equation.

Equations (1.1) and (1.2) are referred to as being doubly nonlinear because of their nonlinearity in both $u$ and $\partial \varphi(u) / \partial x$ [45]. They may be classified as being of second-order parabolic type if $\varphi^{\prime}(u)>0$ and $a_{v}(u, v)>0$ assuming that these derivatives exist, but will degenerate from this type if $\varphi^{\prime}(u)=0$ or $a_{v}(u, v)=0$.

The first significant contribution to the study of equations of the type (1.1) may be attributed to Barenblatt [9]. Investigating self-similar solutions of (1.3) and its two- and three-dimensional analogues, he found explicit solutions of the form

$$
u(x, t)=(t+\tau)^{-1 / p(m+1)} f(\xi) \quad \text { where } \xi=x(t+\tau)^{-1 / p(m+1)}
$$

and

$$
f(\xi)= \begin{cases}{\left[C^{2}-\gamma_{0}^{2}|\xi|^{(p+1) / p}\right]_{+}^{p /(m p-1)}} & \text { if } m p>1, \\ C^{2} \exp \left(-\gamma_{1}^{2}|\xi|^{(p+1) / p}\right) & \text { if } m p=1, \\ \left(C^{2}+\gamma_{0}^{2}|\xi|^{(p+1) / p}\right)^{-p /(1-m p)} & \text { if } m p<1 .\end{cases}
$$


Here $[s]_{+}$denotes $\max \{s, 0\}$. Furthermore, $\tau>0$ and $C>0$ are arbitrary constants, $\gamma_{0}=|m p-1|^{1 / 2}(m(p+1))^{-1 / 2}(p(m+1))^{-1 / 2 p}$ and $\gamma_{1}=p(p+1)^{-(p+1) / 2 p}$.

The booklet [48], published unfortunately in an issue of only 200 copies, provides a good overview of the work of Soviet physicists on equations of the types (1.1) and (1.2) prior to 1982. It comprises an introduction with 75 references and 11 other articles with supplementary references.

A general theory for equations (1.1) and (1.2), that is to say, a study of the existence, uniqueness, regularity and comparison principles for solutions of initialand boundary-value problems of the equation, is as yet not very well developed. The contribution of the 'French school' which was begun in $[\mathbf{4 5}, \mathbf{5 2}]$ is important. A relatively complete list of references to the work of this school can be found in [19] with additional references in $[\mathbf{2 5}, \mathbf{2 6}, \mathbf{4 0}]$.

The Cauchy problem for equation (1.3) with integrable initial data constitutes an exception with respect to the dearth of theory for problems for equations of the types (1.1) and (1.2). See for instance $[25,26,36]$ and the literature cited therein. In $[25,26]$ using the analogy between (1.3) and the porous media equation which was investigated earlier by many authors, Esteban and Vázquez were able to construct a satisfactory theory for the Cauchy problem for (1.3) with integrable initial data. Their definition of a solution seems to be optimal from both a physical and mathematical point of view: solutions satisfy the equation almost everywhere.

The works $[17,19,21,25,26,31,40,48]$ and the references they contain, probably provide the best starting point for an insight into the general theory of equations (1.1) and (1.2). As to particular authors who have contributed to the theory so far, we mention Alt and Luckhaus [1], Atkinson and Bouillet [7, 17], Bamberger [8], Bertsch [10-13], Blanc [14-16], Dal Passo [10, 11, 18], Díaz and de Thelin [19], DiBenedetto [21], van Duijn $[12,22-24]$, Esteban $[12,13,25,26]$, Hilhorst [24], Ivanov [31], Kačur [33, 34], Kalashnikov [35-40], Lions [45], Nagai [47], Pavlov $[48,49]$, Porzio [51], Rykov [53,54], Tsutsumi [57], Vázquez $[25,26]$, Vespri $[51,58-61]$, Yin $[62-64]$ and Zhang $[12,13,22]$ with apologies to those whose names have been omitted.

It may be noted from (1.5) and (1.6), that considering the Cauchy problem for equation (1.3), if $m p>1$ this equation admits a family of explicit solutions possessing an interface separating a region where the solution is positive from one where it is zero. To be explicit, given any $\tau>0$ and $C>0$, there is a solution $u$ such that $u(x, t)>0$ if and only if $|x|<\zeta(t)$ and $t \geqq 0$, where $\zeta$ denotes the interface defined by $\zeta(t):=\left(C / \gamma_{0}\right)^{p /(p+1)}(t+\tau)^{1 / p(m+1)}$. If an equation of type (1.1) or (1.2) admits such a solution, it is said to display finite speed of propagation of perturbations [40]. Thus if $m p>1$, equation (1.3) exhibits this phenomenon. The necessity of the condition $m p>1$ on the parameters in equation (1.3) for this particular equation to admit finite speed of propagation was proved rather later than the discovery of the explicit solution. This was done by Kalashnikov [38] and by Esteban and Vázquez [26]. In the present paper we shall establish a necessary and sufficient condition for equations (1.1) and (1.2) to display finite speed of propagation in their general forms.

The necessary and sufficient condition for equations (1.1) and (1.2) to admit finite speed of propagation may be deduced formally by searching for travelling-wave solutions with bounded support. Suppose that (1.2) admits a solution of the form $u(x, t)=f(\xi)$, where $\xi=x-\lambda t$ and $\lambda$ denotes the wave speed. Then substitution in 
the partial differential equation yields

$$
\left(a\left(f,(\varphi(f))^{\prime}\right)+\lambda f\right)^{\prime}+b\left(f,(\varphi(f))^{\prime}\right)=0 .
$$

Whence, defining $g(\xi):=-(\varphi(f))^{\prime}(\xi)$, one obtains the system

$$
\left\{\begin{array}{l}
f^{\prime}=-g / \varphi^{\prime}(f), \\
(a(f,-g)+\lambda f)^{\prime}=-b(f,-g) .
\end{array}\right.
$$

In the phase-plane the trajectories of this system are given by

$$
\frac{d(a(f,-g)+\lambda f)}{d f}=\frac{b(f,-g) \varphi^{\prime}(f)}{g} .
$$

If now the travelling-wave solution $u$ possesses an interface $\zeta$ such that $u(x, t)>0$ for $x<\zeta(t)$ and $u(x, t)=0$ for $x \geqq \zeta(t)$, necessarily $\zeta(t)=\xi_{0}+\lambda t$ for some $\xi_{0}$, and continuity of the variables $f$ and $g$ implies $f(\xi)>0$ for $\xi<\xi_{0}$ and $f(\xi)=g(\xi)=0$ for $\xi \geqq \xi_{0}$. Consequently, a travelling-wave solution of (1.2) of the suggested type is represented by a trajectory in the half-plane $f>0$ which approaches the point $(f, g)=$ $(0,0)$. Identifying $f$ and $g$ with $s$ and $\theta$ respectively, such a trajectory is equivalent to a solution of

$$
(a(s,-\theta(s))+\lambda s)^{\prime}=\frac{b(s,-\theta(s)) \varphi^{\prime}(s)}{\theta(s)} \text { for } s>0,
$$

with

$$
\theta(0)=0 .
$$

By inverting the first component of (1.7) as

$$
\left(\frac{\varphi^{\prime}}{\theta}\right)(f(\xi)) f^{\prime}(\xi)=-1 \quad \text { for } \xi<\xi_{0},
$$

and coupling this with

$$
f\left(\xi_{0}\right)=0,
$$

it is also feasible to retrace the above argument. The inference is that equation (1.2) has a travelling-wave solution whose support is bounded above if and only if (1.8), (1.9) has a solution $\theta$ for which (1.10), (1.11) is solvable. For this purpose (1.1) may be viewed as a special case of (1.2).

The essence of our main result is the conclusion that (1.1) or (1.2) admits finite speed of propagation if and only if it possesses a travelling-wave solution which exhibits this phenomenon. The proof may be outlined as follows. Let $u$ be a given solution of the equation with initial data $u_{0}$ with compact support and $\zeta_{0}:=\sup \left\{x: u_{0}(x)>0\right\}$. Then, should the partial differential equation admit a travelling-wave solution with bounded support, suitably shifting this wave and applying a comparison principle argument, it can be shown that the wave must majorise $u$ in the domain $x>\zeta_{0}$. Whence since the wave has bounded support, so also does $u$. The key to the alternative case, that the partial differential equation has no travellingwave solution with bounded support, is to observe that when (1.9) is replaced by

$$
\theta(0)=\varepsilon
$$


for $\varepsilon>0$, the inherent singularities in the equations (1.8) and (1.10) are removed. This implies that for any $\xi_{0}, \lambda$ and $\varepsilon>0$, the problems (1.8), (1.12) and (1.10), (1.11) are solvable. Subsequently, repeating the construction of the travelling wave described in the previous paragraph, a continuous function $v(x, t)$, which is positive and a solution of (1.2) for $x<\zeta(t):=\xi_{0}+\lambda t$, which is zero for $x \geqq \zeta(t)$, and which has the property that $(\partial(\varphi(v)) / \partial x)(\zeta(t)-, t)=-\varepsilon<(\partial(\varphi(v)) / \partial x)(\zeta(t)+, t)=0$, may be defined. This means that $v$ is a subsolution of the equation and of travelling-wave type, but not a solution. In the limit $\varepsilon \downarrow 0$ moreover, $v$ must vanish, for otherwise there would be a limit function which would constitute a travelling-wave solution of the equation. To prove then that there is no finite speed of propagation in the open case, the strategy is to demonstrate that it is possible to find a $\xi_{0}$ such that for any wave speed $\lambda$ an $\varepsilon>0$ may be chosen so small that $u$ can be shown to majorise the strict subsolution $v$ of the equation in the domain $x>\xi_{0}$, again using a comparison principle argument. This yields $u(x, t)>0$ for all $\xi_{0}<x<\xi_{0}+\lambda t$. Whence considering the arbitrariness of the wave speed $\lambda$, one can conclude $u(x, t)>0$ for all $x>\xi_{0}$ and $t>0$.

Combining the above arguments, it follows formally that equations (1.1) and (1.2) admit finite speed of propagation if and only if there is a solution $\theta$ to problem (1.8), (1.9) such that (1.10), (1.11) is solvable. By reformulating problems (1.8), (1.9) and (1.8), (1.12) as integral equations, converting the solvability of (1.10), (1.11) into an integrability constraint on the solution $\theta$ of the integral equation, and specifying precisely the comparison principle arguments as local arguments, we shall establish this necessary and sufficient criterion for finite speed of propagation with rigour in the coming sections. In the final section, we discuss the application of the general result to concrete examples of equations (1.1) and (1.2).

\section{Definitions and results}

To prevent any ambiguity, we shall consider non-negative solutions of equations (1.1) and (1.2) only in a half-strip

$$
H:=(0, \infty) \times(0, T], \quad \text { with } 0<T<\infty,
$$

and investigate the possible existence of an interface demarcating the upper boundary of their support. Naturally any solution of a Cauchy problem is also a solution in such a half-strip, and by a simple transformation of the $x$-coordinate any lower boundary of the support of a solution may be transferred into an upper boundary of the support of a solution for an equivalent equation of the form (1.1) or (1.2).

Let $Q$ denote a domain of the form

$$
Q=\left(\eta_{1}, \eta_{2}\right) \times\left(\tau_{1}, \tau_{2}\right], \quad \text { with }-\infty \leqq \eta_{1}<\eta_{2} \leqq \infty, \quad 0 \leqq \tau_{1}<\tau_{2}<\infty,
$$

and consider the equation

$$
\frac{\partial u}{\partial t}=\frac{\partial}{\partial x}\left(a\left(u, \frac{\partial \varphi(u)}{\partial x}\right)\right)+b\left(u, \frac{\partial \varphi(u)}{\partial x}\right)+f
$$

with $f$ in $L^{1}(Q)$.

Definition 2.1. A function $u(x, t)$ is said to be a weak subsolution of equation (2.2) in $Q$ if it is defined, continuous, non-negative and bounded in $\bar{Q}$, the generalised derivative 
$\partial \varphi(u) / \partial x$ exists and is essentially bounded in $Q, b(u, \partial \varphi(u) / \partial x) \in L^{1}(R)$ and

$$
\begin{aligned}
\int_{\mathrm{R}} \int\left\{a\left(u, \frac{\partial \varphi(u)}{\partial x}\right)\right. & \left.\frac{\partial F}{\partial x}-b\left(u, \frac{\partial \varphi(u)}{\partial x}\right) F-f F-u \frac{\partial F}{\partial t}\right\} d x d t \\
+ & \int_{x_{1}}^{x_{2}}\left\{u\left(x, t_{2}\right) F\left(x, t_{2}\right)-u\left(x, t_{1}\right) F\left(x, t_{2}\right)\right\} d x \leqq 0,
\end{aligned}
$$

for all nonempty bounded rectangles $R:=\left(x_{1}, x_{2}\right) \times\left(t_{1}, t_{2}\right] \subseteq Q$ and non-negative functions $F \in C^{1}(\bar{R})$ such that $F\left(x_{1}, t\right)=F_{x}\left(x_{1}, t\right)=F\left(x_{2}, t\right)=F_{x}\left(x_{2}, t\right)=0$ for all $t \in\left[t_{1}, t_{2}\right]$. A weak subsolution is defined to be a weak solution if (2.3) holds with equality for all such $R$ and $F$, and a weak strict subsolution otherwise.

Mutatis mutandis, a weak solution of equation (1.1) or (1.2) in $H$ is a weak solution of (2.2) with $f \equiv 0$ in $H$.

DEFINITION 2.2. We shall say that equations (1.1) and (1.2) display finite speed of propagation if, given any weak solution $u$ in a domain of the type $H$ such that $u_{0}(\cdot):=u(\cdot, 0)$ has compact support and is not identically zero, i.e. setting

$$
\zeta(t):=\sup \{x \in(0, \infty): u(x, t)>0\},
$$

there holds $0<\zeta(0)<\infty$, the solution possesses the property that there is an $X$ and a $\tau \in(0, T]$ such that $\zeta(t) \leqq X<\infty$ for all $t \in[0, \tau]$.

In this case $\zeta$ constitutes an interface, front, or free boundary in the solution.

A weak solution $u$ of equation (1.1) or (1.2) in a domain $Q$ is generally referred to as a strong solution if the generalised derivative $\partial u / \partial t$ exists and is integrable in $Q$. It is called the limit of strong solutions if there exists a sequence of functions $\left\{f_{k}\right\}_{k=1}^{\infty}$ such that $f_{k} \rightarrow f$ as $k \rightarrow \infty$ and each equation (2.2) with $f$ replaced by $f_{k}$ has a strong solution $u_{k}$ with the property that $u_{k} \rightarrow u$ as $k \rightarrow \infty$ in a suitable sense. From the results of $[\mathbf{8}]$, it follows that if equation (2.2) has the form

$$
\frac{\partial u}{\partial t}=\frac{\partial}{\partial x}\left(\left|\frac{\partial u^{m}}{\partial x}\right|^{p-1} \frac{\partial u^{m}}{\partial x}\right)+f
$$

with $m>1$ and $p>0$, and if $f$ is non-negative and satisfies certain continuity assumptions, given a suitable continuous initial data function with compact support in $\left(\eta_{1}, \eta_{2}\right)$ for the first boundary-value problem with homogeneous Dirichlet boundary data in a bounded domain $Q$ of the type (2.1), there is a unique weak solution to this problem which is the limit of strong solutions. Whilst, from the results of [19], if (2.2) has the form

$$
\frac{\partial u}{\partial t}=\frac{\partial}{\partial x}\left(\left|\frac{\partial \varphi(u)}{\partial x}+\psi(u)\right|^{p-1}\left(\frac{\partial \varphi(u)}{\partial x}+\psi(u)\right)\right)+c(u)+f
$$

where $p>0$, if the function $\varphi$ satisfies Hypothesis 1.1(i), and if the functions $\psi, c$ and $f \in L^{1}\left(\tau_{1}, \tau_{2} ; L^{\infty}\left(\eta_{1}, \eta_{2}\right)\right)$ satisfy certain continuity and growth conditions, it is also known that the stated problem has a unique strong solution. Moreover, in both cases, given any two solutions $u^{(1)}$ and $u^{(2)}$ with corresponding functions $f^{(1)}$ and $f^{(2)}$ such that $f^{(1)} \leqq f^{(2)}$ almost everywhere in $Q$ and $u^{(1)} \leqq u^{(2)}$ everywhere on $\bar{Q} \backslash Q$ there holds $u^{(1)} \leqq u^{(2)}$ everywhere in $\bar{Q}$. Variants on this comparison principle for other 
equations of the same ilk can be found in $[1,17,22,24,37,47,49,57]$, in particular for equation (1.1) in bounded and unbounded domains in [17], and for the equation

$$
\frac{\partial u}{\partial t}=\frac{\partial}{\partial x}\left(a\left(u, \frac{\partial \varphi(u)}{\partial x}\right)\right)+c(u)
$$

in [1]. Noting that any weak solution of equation (2.2) with $f \leqq 0$ almost everywhere in a domain $Q$ is a weak subsolution of (1.2) in $Q$, and a weak strict subsolution if $f<0$ in some subset of $Q$ of positive measure, we shall suppose that these results are generalisable as follows.

Hypothesis 2.3 (Comparison principle). Let $u$ denote a weak solution of equation (1.1) or (1.2) in $H$ and let $Q$ be a domain of the form (2.1) such that $Q \subseteq H$.

(i) Given any weak solution $v$ of (1.1) or (1.2), respectively, in $Q$ such that $u(x, t) \leqq v(x, t)$ for all $(x, t) \in \bar{Q} \backslash Q$, there holds $u(x, t) \leqq v(x, t)$ for all $(x, t) \in \bar{Q}$.

(ii) Given any weak strict subsolution $v$ of (1.1) or (1.2), respectively, in $Q$ such that $v(x, t) \leqq u(x, t)$ for all $(x, t) \in \bar{Q} \backslash Q$, there holds $v(x, t) \leqq u(x, t)$ for all $(x, t) \in \bar{Q}$.

With the comparison principle as a tool, we shall establish the necessary and sufficient condition for equations of the types (1.1) and (1.2) to admit finite speed of propagation. We formulate the result in the most general form which includes the indicated cases of physical interest.

THEOREM 2.4. Equation (1.1) displays finite speed of propagation if and only if there is a real parameter $\lambda$ such that the equation

$$
a(s,-\theta(s))+\lambda s=0
$$

has a non-negative continuous solution $\theta$ in an interval $[0, \delta)$ satisfying

$$
\int_{0}^{\delta} \frac{\varphi^{\prime}(s)}{\theta(s)} d s<\infty \quad \text { for some } 0<\delta<\infty .
$$

Equation (1.2) displays finite speed of propagation if and only if there is a real parameter $\lambda$ such that the equation

$$
a(s,-\theta(s))+\lambda s=\int_{0}^{s} \frac{b(r,-\theta(r)) \varphi^{\prime}(r)}{\theta(r)} d r
$$

has a non-negative continuous solution $\theta$ in an interval $[0, \delta)$ satisfying (2.6).

For equation (1.3), the equation (2.5) reduces simply to $-|\theta(s)|^{p-1} \theta(s)+\lambda s=0$. Hence necessarily $\lambda \geqq 0$ for a non-negative solution which needs to be $\theta(s)=(\lambda s)^{1 / p}$. The theorem then states that (1.3) displays finite speed of propagation if and only if there is a $\lambda \geqq 0$ such that

$$
\int_{0}^{\delta} \frac{m s^{m-1}}{(\lambda s)^{1 / p}} d s<\infty \quad \text { for some } 0<\delta<\infty .
$$

This condition is plainly satisfied for all $\lambda>0$ if $m p>1$ and no $\lambda \geqq 0$ if $m p \leqq 1$. Thus the theorem readily yields the known condition $m p>1$ as necessary and sufficient for finite speed of propagation by the prototypical equation (1.3).

In the last section of this paper we shall discuss further applications of the above 
theorem and their relation to earlier results on finite speed of propagation for particular equations of the types (1.1) and (1.2).

Note though that the criterion for finite speed of propagation provided by Theorem 2.4 is independent of the specific formulation of the equation involved. Two equations of the type (1.1) with coefficients $a, \varphi$ and $\tilde{a}, \tilde{\varphi}$ are formally equivalent if $\tilde{a}\left(u, \tilde{\varphi}^{\prime}(u) w\right)=a\left(u, \varphi^{\prime}(u) w\right)$ for all $(u, w) \in \mathbb{R}^{+} \times \mathbb{R}$, whilst two equations of the form (1.2) with coefficients $a, b, \varphi$ and $\tilde{a}, \tilde{b}, \tilde{\varphi}$ are correspondingly equivalent if $\quad \tilde{a}\left(u, \tilde{\varphi}^{\prime}(u) w\right)-\tilde{a}(u, 0)=a\left(u, \varphi^{\prime}(u) w\right)-a(u, 0) \quad$ and $\quad \tilde{b}\left(u, \tilde{\varphi}^{\prime}(u) w\right)+\tilde{a}_{u}(u, 0) w=$ $b\left(u, \varphi^{\prime}(u) w\right)+a_{u}(u, 0) w$ for all $(u, w) \in \mathbb{R}^{+} \times \mathbb{R}$. However, in both cases the criterion for finite speed of propagation can be seen to be identical by observing that if $\theta$ and $\tilde{\theta}$ denote the appropriate sought-for functions in Theorem 2.4, they can be identified via $\tilde{\varphi}^{\prime}(s) / \tilde{\theta}(s)=\varphi^{\prime}(s) / \theta(s)$.

\section{Proof of main theorem}

Before proving Theorem 2.4 we should for clarity indicate what we mean by a solution of the integral equation (2.7). For this purpose, we consider the extension

$$
a(s,-\theta(s))+\lambda s=a(0,-\varepsilon)+\int_{0}^{s} \frac{b(r,-\theta(r)) \varphi^{\prime}(r)}{\theta(r)} d r,
$$

where $\varepsilon \geqq 0$ is a real parameter. To avoid ambiguity in the integrand in (3.1), we define

$$
I(r, \theta)= \begin{cases}b(r,-\theta) \varphi^{\prime}(r) / \theta & \text { if } b(r,-\theta) \varphi^{\prime}(r) \neq 0 \text { or } \theta \neq 0, \\ -b_{v}(r, 0-) \varphi^{\prime}(r) & \text { if } b(r,-\theta) \varphi^{\prime}(r)=0 \text { and } \theta=0 .\end{cases}
$$

DEFINITION 3.1. A function $\theta$ is said to be a solution of (3.1) if it is defined non-negative and continuous in a neighbourhood $[0, \delta), I(r, \theta(r)) \in L^{1}(0, \delta)$, and

$$
a(s,-\theta(s))+\lambda s=a(0,-\varepsilon)+\int_{0}^{s} I(r, \theta(r)) d r \quad \text { for all } s \in[0, \delta),
$$

for some $0<\delta<\infty$.

A solution of equation (2.7) is a solution of (3.1) with $\varepsilon=0$. From the above definition of a solution $\theta$ of (3.1), it follows that $a(0,-\theta(0))=a(0,-\varepsilon)$ and thus, by the monotonicity of $a$ embodied in Hypothesis 1.1(ii), that $\theta(0)=\varepsilon$. In particular, this means that for a solution of $(2.7)$ there holds $\theta(0)=0$.

We can now directly establish the sufficiency of the existence of a solution of $(2.7)$ satisfying (2.6) for finite speed of propagation for equation (1.2).

Suppose that there is a $\lambda$ such that (2.7) has a solution $\theta$ on some interval $[0, \delta)$ satisfying (2.6). Let $u$ denote a solution of equation (1.2) in some domain $H$, and suppose that $\zeta(0)<\infty$, where $\zeta$ is defined by (2.4). Subsequently, choose a point $x_{1}$ such that

$$
\zeta(0)<x_{1}<\zeta(0)+\int_{0}^{\delta} \frac{\varphi^{\prime}(s)}{\theta(s)} d s
$$


In this event, there exists a $\tau \in(0, T]$ such that the function $v$ given by

$$
\int_{0}^{v(x, t)} \frac{\varphi^{\prime}(s)}{\theta(s)} d s=\max \left\{x_{1}-x+\lambda t, 0\right\}
$$

is defined and continuous in the closure of the domain $Q:=(\zeta(0), \infty) \times(0, \tau]$. Furthermore, since $v(\zeta(0), 0)>0=u(\zeta(0), 0)$, by a continuity argument one may choose $\tau$ so small that

$$
v(\zeta(0), t)>u(\zeta(0), t) \text { for all } t \in[0, \tau] .
$$

Subsequently, it can be verified (cf. [30]) that $v$ is a weak solution of (1.2) of travelling-wave type in the domain $Q$. Besides (3.4), one has $v(x, 0) \geqq 0=u(x, 0)$ for all $x \in[\zeta(0), \infty)$. So, by the comparison principle of Hypothesis 2.3(i), there holds $v(x, t) \geqq u(x, t)$ for all $(x, t) \in \bar{Q}$. This gives $\zeta(t) \leqq x_{1}+\lambda t$ for all $t \in[0, \tau]$.

To prove the necessity of the existence of a solution to (2.7) satisfying (2.6) for finite speed of propagation by equation (1.2), we shall use the following result whose proof we postpone to the next section.

LEMMA 3.2. For all $\lambda$ and all $\varepsilon>0$, equation (3.1) admits a maximal solution $\theta(s ; \lambda, \varepsilon)$ on a maximal interval of existence $[0, M(\lambda, \varepsilon))$.

Since by definition $\theta(0 ; \lambda, \varepsilon)=\varepsilon$, it follows from the continuity of $\theta(s ; \lambda, \varepsilon)$ that for any $\lambda$ and $\varepsilon>0$ we may define

$$
\mu(\lambda, \varepsilon):=\sup \left\{s \in(0, M(\lambda, \varepsilon)): \int_{0}^{s} \frac{\varphi^{\prime}(r)}{\theta(r ; \lambda, \varepsilon)} d r<\infty\right\}>0 .
$$

Moreover, the following may be said about this variable.

LEMMA 3.3. Let $\lambda$ be such that equation (2.7) has no solution satisfying (2.6). Then: either

$$
\mu(\lambda, \varepsilon) \rightarrow 0 \quad \text { as } \varepsilon \downarrow 0,
$$

in which case for all sufficiently small $\&$ there holds

$$
\theta(s ; \lambda, \varepsilon) \rightarrow 0 \quad \text { as } s \uparrow \mu(\lambda, \varepsilon)<\infty
$$

and

$$
b(\mu(\lambda, \varepsilon), 0) \geqq 0
$$

or there exists a $v \leqq \infty$ such that

$$
\mu(\lambda, \varepsilon) \rightarrow v>0 \quad \text { as } \varepsilon \downarrow 0,
$$

in which case

$$
\int_{0}^{\delta} \frac{\varphi^{\prime}(s)}{\theta(s ; \lambda, \varepsilon)} d s \rightarrow \infty \quad \text { as } \varepsilon \downarrow 0
$$

for all $\delta \in(0, v)$.

Like the proof of Lemma 3.2, we defer the verification of this lemma until the next section. 
Suppose now that $u$ is a solution of equation (1.2) in $H$ with $u_{0}(\cdot):=u(\cdot, 0)$ not identically zero. Then by the continuity of $u$ there exists a $\tau \in(0, T]$ such that $u(\cdot, t)$ is not identically zero for every $t \in[0, \tau]$. Pick an arbitrary $t_{1} \in(0, \tau]$ and $x_{0} \in(0, \infty)$ such that $u\left(x_{0}, t_{1}\right)>0$. Subsequently, choose a $\kappa$ and a $t_{0} \in\left[0, t_{1}\right)$ for which

$$
u\left(x_{0}, t\right) \geqq \kappa>0 \text { for all } t \in\left[t_{0}, t_{1}\right]
$$

and let

$$
Q:=\left(x_{0}, \infty\right) \times\left(t_{0}, t_{1}\right] .
$$

Finally, let $\lambda$ and $\varepsilon>0$ be arbitrary and consider the function $v$ defined by

$$
\int_{0}^{v(x, t)} \frac{\varphi^{\prime}(s)}{\theta(s ; \lambda, \varepsilon)} d s=\max \left\{x_{0}-x+\lambda\left(t-t_{0}\right), 0\right\} \quad \text { for } x>x_{0}+\lambda\left(t-t_{0}\right)-\eta
$$

and

$$
v(x, t)=\mu(\lambda, \varepsilon) \text { for } x \leqq x_{0}+\lambda\left(t-t_{0}\right)-\eta,
$$

where

$$
\eta=\int_{0}^{\mu(\lambda, \varepsilon)} \frac{\varphi^{\prime}(s)}{\theta(s ; \lambda, \varepsilon)} d s .
$$

We assert that if (2.7) has no solution satisfying (2.6) for a fixed $\lambda$, we can pick an $\varepsilon$ so small that $v$ is defined in $\bar{Q}$, and

$$
v\left(x_{0}, t\right)<\kappa \text { for all } t \in\left[t_{0}, t_{1}\right] .
$$

If $v$ is defined in $\bar{Q}$, following the work $[30]$ it can be shown that $v$ is a weak strict subsolution of (1.2) in $Q$. (Note that in contrast to the function defined by (3.3), the present $v$ is not a weak solution of (1.2) in $Q$ because the function $\theta$ which was previously a solution of (2.7) has been replaced by a solution of (3.1) with an $\varepsilon>0$. Nonetheless $v$ is a weak subsolution of (1.2). Formally, the function $v$ is a solution of (2.2) in the sense of distributions with

$$
f= \begin{cases}-b(u(\mu, \varepsilon), 0) & \text { for } x \leqq x_{0}+\lambda\left(t-t_{0}\right)-\eta \\ -\varepsilon \delta\left(x-x_{0}-\lambda\left(t-t_{0}\right)\right) & \text { for } x>x_{0}+\lambda\left(t-\eta_{0}\right)-\eta\end{cases}
$$

where $\delta$ denotes the Dirac-delta function in $\mathbb{R}$.) Subsequently, since (3.11) and (3.15) hold, and $v\left(x, t_{0}\right)=0 \leqq u\left(x, t_{0}\right)$ for all $x \in\left[x_{0}, \infty\right)$, by the comparison principle of Hypothesis 2.3(ii), we deduce that $v(x, t) \leqq u(x, t)$ for all $(x, t) \in \bar{Q}$. This implies $u(x, t)>0$ for all $x_{0}<x<x_{0}+\lambda\left(t-t_{0}\right)$ and $t_{0}<t \leqq t_{1}$, whence, since $\lambda$ was arbitrary, $u(x, t)>0$ for all $(x, t) \in Q$. Thus equation (1.2) does not admit finite speed of propagation.

To prove that if there is no $\lambda$ for which (2.7) has a solution satisfying (2.6), then (1.2) does not admit finite speed of propagation, it therefore suffices to show that if the integral equation (2.7) has no solution satisfying (2.6) for any fixed $\lambda$, we can find an $\varepsilon>0$ such that the function $v$ given by (3.12)-(3.14) is defined in $\bar{Q}$ and satisfies (3.15). To do this, we distinguish two cases according to the alternatives stated in Lemma 3.3. In the first case, viz $\mu(\lambda, \varepsilon) \rightarrow 0$ as $\varepsilon \downarrow 0$, we can choose an $\varepsilon>0$ 
so small that $\mu(\lambda, \varepsilon)<\kappa$. With such a choice, $v$ is automatically defined in $\bar{Q}$ by (3.12)-(3.14) and satisfies (3.15). On the other hand, if there is a $v \leqq \infty$ such that (3.9) holds, we may fix a real number $\delta \in(0, \min \{v, \kappa\})$ and by Lemma 3.3 choose an $\varepsilon>0$ so small that $\mu(\lambda, \varepsilon)>\delta$ and

$$
\int_{0}^{\delta} \frac{\varphi^{\prime}(s)}{\theta(s ; \lambda, \varepsilon)} d s>\lambda\left(t_{1}-t_{0}\right) .
$$

This guarantees that $\bar{Q} \subseteq\left\{(x, t): x>x_{0}+\lambda\left(t-t_{0}\right)-\eta\right.$ and $\left.t_{0} \leqq t \leqq t_{1}\right\}$ where $\eta$ is defined by (3.14) and also that $v\left(x_{0}, t\right)<\delta<\kappa$ for all $t \in\left[t_{0}, t_{1}\right]$. So in this case also, the assertion is verified.

The proof of the part of Theorem 2.4 relating to equation (1.1) may be realised by suitably editing the above argument for equation (1.2).

From the above proof of Theorem 2.4, it follows that we can actually state the following results.

Corollary 3.4. Suppose that $\varphi$ satisfies Hypothesis 1.1(i), a satisfies Hypothesis 1.1(ii), and $b \in C\left(\mathbb{R}^{+} \times \mathbb{R}\right)$ with $b(0,0)=0$. Suppose furthermore that Hypothesis 2.3(i) holds. Let $u$ be an arbitrary weak solution of equation (1.1) or (1.2) in $H$ such that $\zeta(0)<\infty$ where $\zeta$ is given by (2.4). Then, given any $x_{1}>\zeta(0)$ and $\lambda$ such that (2.5) or (2.7), respectively, has a solution satisfying (2.6), there exists $a \tau \in(0, T]$ such that $\zeta(t) \leqq x_{1}+\lambda t$ for all $t \in[0, \tau]$.

CoRollary 3.5. Supoose that $\varphi$ satisfies Hypothesis 1.1(i), a satisfies Hypothesis $1.1(\mathrm{ii})$, and $b \in C\left(\mathbb{R}^{+} \times \mathbb{R}\right)$ with $b(0,0)=0$. Suppose furthermore that Lemmata 3.2 and 3.3 and Hypothesis 2.3(ii) holds. Let $u$ be an arbitrary weak solution of equation (1.1) or $(1.2)$ in $H$ such that $u_{0}(\cdot):=u(\cdot, 0)$ is not identically zero. Then, if there is no $\lambda$ such that (2.5) or (2.7), respectively, has a solution satisfying (2.6), there exists a $\tau \in(0, T]$ such that the set $\{x \in(0, \infty): u(x, t)>0\}$ is nonempty, connected and unbounded for all $t \in(0, \tau]$.

It transpires thus that the extra conditions in Hypothesis 1.2 are intended for the verification of Lemmata 3.2 and 3.3. The stated conditions are sufficient but not the optimal assumptions for this task.

Note that Corollary 3.4 yields the continuity from the right of the interface $\zeta$ defined by (2.4).

\section{Theory of the integral equation}

In this section we shall set up a theory for the equation (2.5) and the integral equation (2.7) with as goals proving Lemmata 3.2 and 3.3 and providing a little more insight into the criterion for finite speed of propagation. Assuming that Hypothesis 1.2 holds, or that $b \equiv 0$ and Hypothesis 1.1 holds, our main results on the equation (2.7) are the following:

Proposition 4.1. For any $\lambda$ and any $\varepsilon>0$, equation (3.1) has a solution.

Proposition 4.2. For any $\lambda$ and any $\varepsilon \geqq 0$, equation (3.1) has no solution, or it has a unique solution, or it has an uncountable number of solutions among which there is a maximal solution. 
Proposition 4.3. For any $\lambda$ and any $\varepsilon \geqq 0$, given any solution $\theta$ of (3.1), this solution is extensible to a maximal interval of existence $[0, M)$ such that $\theta(s) \rightarrow \infty$ as $s \uparrow M$, $M=\infty$, or, $\theta(s) \rightarrow 0$ as $s \uparrow M$.

Consequently, for any $\lambda$ and every $\varepsilon>0$, we may denote by $\theta(s ; \lambda, \varepsilon)$ the maximal (or unique) solution of $(3.1)$ with $M(\lambda, \varepsilon)$ the supremum of its maximal interval of existence.

Proposition 4.4. For any $\lambda$, there exists an $E(\lambda), 0 \leqq E(\lambda) \leqq \infty$, with the following properties:

(i) $E\left(\lambda_{1}\right) \geqq E\left(\lambda_{2}\right)$ for every $\lambda_{1} \leqq \lambda_{2}$;

(ii) $M\left(\lambda_{1}, \varepsilon_{1}\right) \geqq M\left(\lambda_{2}, \varepsilon_{2}\right)$ and $\theta\left(s ; \lambda_{1}, \varepsilon_{1}\right) \leqq \theta\left(s ; \lambda_{2}, \varepsilon_{2}\right)$ for all $s \in\left[0, M\left(\lambda_{2}, \varepsilon_{2}\right)\right)$, for every $\lambda_{1} \leqq \lambda_{2}$ and $E\left(\lambda_{1}\right)<\varepsilon_{1}<\varepsilon_{2}<\infty$;

(iii) $M\left(\lambda_{1}, \varepsilon_{1}\right) \leqq M\left(\lambda_{2}, \varepsilon_{2}\right)$ and $\theta\left(s ; \lambda_{1}, \varepsilon_{1}\right) \leqq \theta\left(s ; \lambda_{2}, \varepsilon_{2}\right)$ for all $s \in\left[0, M\left(\lambda_{1}, \varepsilon_{1}\right)\right)$, for every $\lambda_{1} \leqq \lambda_{2}$ and $0<\varepsilon_{1}<\varepsilon_{2} \leqq E\left(\lambda_{2}\right)$.

This proposition implies that for each $\lambda$ we may define

$$
N(\lambda):=\lim _{\varepsilon \downarrow 0} M(\lambda ; \varepsilon)
$$

and

$$
\theta(s ; \lambda, 0):=\lim _{\varepsilon \downarrow 0} \theta(s ; \lambda, \varepsilon)
$$

for all $s \in[0, N(\lambda))$. Moreover,

$\theta\left(s ; \lambda_{1}, 0\right) \leqq \theta\left(s ; \lambda_{2}, 0\right)$ for all $s \in\left[0, \min \left\{N\left(\lambda_{1}\right), N\left(\lambda_{2}\right)\right\}\right]$ for every $\lambda_{1} \leqq \lambda_{2}$.

Proposition 4.5. If equation (2.7) has a solution for some values $\lambda^{*}$, then for every $\lambda \geqq \lambda^{*}$ there holds $N(\lambda)>0$, and $\theta(s ; \lambda, 0)$ defines the maximal solution of $(2.7)$ on a maximal interval of existence $[0, M(\lambda, 0))$ with $0<M(\lambda, 0) \leqq N(\lambda)$.

Propositions 4.1 to 4.5 are simplest to check if $b \equiv 0$, in which case (2.7) reduces to (2.5). Let

$$
\Omega=\left\{(u, a(u, v)):(u, v) \in \overline{\mathbb{R}^{+}} \times \mathbb{R}\right\}
$$

and define the function $A$ on $\Omega$ by

$$
A(u, a(u, v))=v \quad \text { for all }(u, v) \in \overline{\mathbb{R}^{+}} \times \mathbb{R} .
$$

By Hypothesis 1.1(ii), $A$ is continuous in $\Omega, A(0,0)=0$ and $A\left(u, w_{1}\right)>A\left(u, w_{2}\right)$ for all $\left(u, w_{1}\right),\left(u, w_{2}\right) \in \Omega$ with $w_{1}>w_{2}$. Consequently, equation (3.1) can be expressed simply as $\theta(s)=-A(s, a(0,-\varepsilon)-\lambda s)$. In this case, it is not too hard to see that (3.1) has a unique solution for any $\lambda$ and any $\varepsilon>0$, and that this solution is defined nonnegative and continuous as long as $(s, a(0,-\varepsilon)-\lambda s) \in \Omega$ and $A(s, a(0,-\varepsilon)-\lambda s) \leqq 0$. Thus we can state simply that

$$
\theta(s ; \lambda, \varepsilon)=-A(s, a(0,-\varepsilon)-\lambda s),
$$

with

$$
\begin{gathered}
M(\lambda, \varepsilon)=\sup \left\{s \in \mathbb{R}^{+}:(r, a(0,-\varepsilon)-\lambda r) \in \Omega \text { and } A(r, a(0,-\varepsilon)-\lambda r) \leqq 0\right. \\
\text { for all } r \in[0, s)\}
\end{gathered}
$$


for any $\lambda$ and any $\varepsilon>0$, and define $\theta(s ; \lambda, 0)$ and $M(\lambda, 0)$ analogously. If $M(\lambda, \varepsilon)<\infty$, then in view of the properties of $A$ either $(M(\lambda, \varepsilon), a(0,-\varepsilon)-\lambda M(\lambda, \varepsilon)) \in \partial \Omega$ in which case $\theta(s ; \lambda, \varepsilon) \rightarrow \infty$ as $s \uparrow M(\lambda, \varepsilon)$, or $A(M(\lambda, \varepsilon), a(0,-\varepsilon)-\lambda M(\lambda, \varepsilon))=0$ in which case $\theta(s ; \lambda, \varepsilon) \rightarrow 0$ as $s \uparrow M(\lambda, \varepsilon)$. This vindicates Propositions 4.1 to 4.3 when $b \equiv 0$. Furthermore, defining

$$
E(\lambda):=\inf \{\varepsilon>0: M(\lambda, \varepsilon)<\infty \text { and }(M(\lambda, \varepsilon), a(0,-\varepsilon)-\lambda M(\lambda, \varepsilon)) \in \partial \Omega\}
$$

if this set is nonempty and $E(\lambda):=\infty$ otherwise, Propositions 4.4 and 4.5 are also readily checked in this case.

When $b \neq 0$, equation (3.1) may be treated using a combination of the analysis above and that applied in [28] for the integral equation

$$
\theta(s)=f(s)+\int_{0}^{s} \frac{g(r)}{\theta(r)} d r
$$

where $f \in C\left(\overline{\mathbb{R}^{+}}\right)$with $f(0) \geqq 0$ and $g \in L^{1}(0, s)$ for all $s \in \mathbb{R}^{+}$. Letting $\Omega$ denote the set defined by (4.3) and $A$ the function defined on $\Omega$ by (4.4), we may rewrite (3.1) as

$$
\theta(s)=-A\left(s, a(0,-\varepsilon)-\lambda s+\int_{0}^{s} \frac{b(r,-\theta(r)) \varphi^{\prime}(r)}{\theta(r)} d r\right) .
$$

In view of Hypothesis 1.2 , it can be shown that for any $\left(s^{*}, \rho\right),\left(s^{*}, \rho\right)$, $\left(s^{*}, \bar{\rho}\right) \in \overline{\mathbb{R}^{+}} \times \mathbb{R}^{+}$such that $\rho<\rho<\bar{\rho}$ and $A\left(s^{*}, a\left(s^{*},-\underline{\rho}\right)\right)<0$, it is possible to find a $\gamma$ for which the mapping defined by

$$
\theta \rightarrow-A\left(s, a\left(s^{*},-\rho\right)-\lambda\left(s-s^{*}\right)+\int_{s^{*}}^{s} \frac{b(r,-\theta(r)) \varphi^{\prime}(r)}{\theta(r)} d r\right)
$$

is a contraction on the set of functions $\theta$ defined on $\left[\max \left\{0, s^{*}-\gamma\right\}, s^{*}+\gamma\right]$ for which $\rho \leqq \theta(s) \leqq \bar{\rho}$ for every $s \in\left[\max \left\{0, s^{*}-\gamma\right\}, s^{*}+\gamma\right]$. Taking $s^{*}=0$ and $\rho=\varepsilon$ gives the existence result embodied in Proposition 4.1. The main conclusions of Propositions 4.2 and 4.3 may subsequently be derived applying the extension argument used for solutions of equation (4.6) in [28]. The only (inessential) complication is that, in contrast to a solution of equation (4.6), for equation (3.1) it is possible that a solution $\theta$ with a bounded maximal interval of existence is unbounded. This possibility could be excluded for (4.6) because of the simpler structure.

Letting $\theta(s ; \lambda, \varepsilon)$ denote the maximal solution of $(3.1)$ with $[0, M(\lambda, \varepsilon))$ its maximal interval of existence for any $\lambda$ and any $\varepsilon>0$, Proposition 4.4 may be obtained by wielding the contraction argument used above in a manner similar to that applied for equation (4.6) in [28]. The critical value $E(\lambda)$ is defined by (4.5) if the stated set is nonempty and by $E(\lambda):=\infty$ otherwise. We note that compared to the equivalent analysis for (4.6), the introduction of this variable is the price we have to pay for being unable to exclude the possibility that solutions with a bounded maximal interval of existence themselves become unbounded. Proposition 4.5 follows immediately.

This substantiates the general theory of equation (2.7). Propositions 4.1 and 4.2 yield Lemma 3.2. Furthermore, Proposition 4.5 and (4.2) have the following noteworthy consequence with regard to the criterion for finite speed of propagation. 
REMARK 4.6. If equation (2.5) or (2.7) has a solution which satisfies (2.6) for some value $\lambda^{*}$ the same can be said for all $\lambda \geqq \lambda^{*}$.

We have however still to complete the proof of Lemma 3.3 to justify our main result on finite speed of propagation.

Proof of Lemma 3.3. By the above, the variable $\mu(\lambda, \varepsilon)$ given by (3.5) is well defined for any $\lambda$ and any $\varepsilon>0$. Moreover, by the continuity of $\theta(s ; \lambda, \varepsilon)$, either $\mu(\lambda, \varepsilon)=$ $M(\lambda, \varepsilon)<\infty$ and $\theta(s ; \lambda, \varepsilon) \rightarrow \infty$ as $s \uparrow \mu(\lambda, \varepsilon), \mu(\lambda, \varepsilon)=M(\lambda, \varepsilon)=\infty$, or $\mu(\lambda, \varepsilon)<\infty$ and $\theta(s ; \lambda, \varepsilon) \rightarrow 0$ as $s \uparrow \mu(\lambda, \varepsilon)$. Subsequently, we may define $\tilde{E}(\lambda)$ with $E(\lambda) \leqq \tilde{E}(\lambda) \leqq \infty$ by $\tilde{E}(\lambda):=\inf \{\varepsilon>0: \mu(\lambda, \varepsilon)<\infty$ and $\theta(s ; \lambda, \varepsilon) \rightarrow \infty$ as $s \uparrow \mu(\lambda, \varepsilon)\}$ if this infimum is taken over a nonempty set and $\tilde{E}(\lambda):=\infty$ otherwise. Proposition 4.4 implies that $\mu\left(\lambda, \varepsilon_{1}\right) \geqq \mu\left(\lambda, \varepsilon_{2}\right)$ and $\theta\left(s ; \lambda, \varepsilon_{1}\right) \leqq \theta\left(s ; \lambda, \varepsilon_{2}\right)$ for all $s \in\left[0, \mu\left(\lambda, \varepsilon_{2}\right)\right)$ for any $\tilde{E}(\lambda)<\varepsilon_{1}<\varepsilon_{2}$, whilst $\mu\left(\lambda, \varepsilon_{1}\right) \leqq \mu\left(\lambda, \varepsilon_{2}\right)$ and $\theta\left(s ; \lambda, \varepsilon_{1}\right) \leqq \theta\left(s ; \lambda, \varepsilon_{2}\right)$ for all $s \in\left[0, \mu\left(\lambda, \varepsilon_{1}\right)\right)$ for any $0<\varepsilon_{1}<\varepsilon_{2} \leqq \tilde{E}(\lambda)$. Whence for every fixed $\lambda$ the variable

$$
v:=\lim _{\varepsilon \downarrow 0} \mu(\lambda, \varepsilon)
$$

is well defined. Supposing that for fixed $\lambda$ equation (2.7) has no solution satisfying (2.6), the two conclusions of Lemma 3.3 can now be distinguished by whether or not $v=0$.

Suppose firstly that $v=0$. Then by definition (3.6) holds. Consequently (3.7) holds for all sufficiently small $\varepsilon$. Let now $\varepsilon$ be as such. Dropping the $\lambda$ and $\varepsilon$ from the notation of $\theta$ and $\mu$ for convenience, from (3.1) and (3.7) it follows that setting

$$
\int_{s}^{\mu} I(r, \theta(r)) d r:=\lim _{w \uparrow \mu} \int_{s}^{w} I(r, \theta(r)) d r \quad \text { for all } s \in[0, \mu),
$$

where $I(r, \theta(r))$ is defined by (3.2), we may let $s \uparrow \mu$ in (3.1) to deduce

$$
a(\mu, 0)+\lambda \mu=a(0,-\varepsilon)+\int_{0}^{\mu} I(r, \theta(r)) d r .
$$

Whence

$$
a(\mu, 0)-a(s,-\theta(s))=-\lambda(\mu-s)+\int_{s}^{\mu} I(r, \theta(r)) d r
$$

for all $s \in[0, \mu)$. Now suppose that $b(\mu, 0)<0$. Then by the continuity of $b, \varphi^{\prime}$ and $\theta$ there exists a $\rho>0$ and an $s_{0} \in(0, \mu)$ such that $I(r, \theta(r))<-\rho / \theta(r)$ for all $r \in\left(s_{0}, \mu\right)$. Substituting in (4.8), this gives

$$
a(\mu, 0)-a(s,-\theta(s)) \leqq-\lambda(\mu-s)-\rho \int_{s}^{\mu} \frac{d r}{\theta(r)} .
$$

However, $a(s,-\theta(s)) \leqq a(s, 0)$ by Hypothesis 1.2 , whilst we may also choose an $s \in\left(s_{0}, \mu\right)$ arbitrarily close to $\mu$ such that $\theta(r) \leqq \theta(s)$ for all $r \in(s, \mu)$. Taking such an $s$ in (4.9) subsequently gives

$$
a(\mu, 0)-a(s, 0) \leqq-\lambda(\mu-s)-\rho(\mu-s) / \theta(s) .
$$


Hence, dividing by $(\mu-s)$ and letting the chosen $s$ approach $\mu$, we obtain

$$
\liminf _{s \uparrow \mu}\{a(\mu, 0)-a(s, 0)\} /(\mu-s)=-\infty .
$$

This contradicts Hypothesis 1.2. Thus necessarily $b(\mu, 0) \geqq 0$. This confirms (3.8).

Suppose on the other hand that $v>0$. Let $\delta \in(0, v)$ and pick $\varepsilon_{0}>0$ so small that $\mu(\lambda, \varepsilon)>\delta$ for all $\varepsilon \in\left(0, \varepsilon_{0}\right]$. Recalling Proposition 4.4 , define $\theta(s ; \lambda, 0)$ by (4.1). We assert that if

$$
\int_{0}^{\delta} \frac{\varphi^{\prime}(s)}{\theta(s ; \lambda, \varepsilon)} d s<L \quad \text { for all } 0<\varepsilon \leqq \varepsilon_{0}
$$

for some $L<\infty$, then $\theta(s ; \lambda, 0)$ is a solution of $(2.7)$ on $[0, \delta)$ which satisfies (2.6). Thus, since $\lambda$ is such that (2.7) has no such solution, (4.10) cannot be true, and (3.10) must hold.

To prove the above assertion, let $\Theta<\infty$ be such that $\theta(s ; \lambda, \varepsilon) \leqq \Theta$ for all $0 \leqq s \leqq \delta$ and $0 \leqq \varepsilon \leqq \varepsilon_{0}$; and, in the light of Hypothesis 1.2 , let $B \in L^{1}(0, \delta)$ be such that $\left|b_{v}(u, v) \varphi^{\prime}(u)\right| \leqq B(u)$ for all $0<u<\delta$ and $0 \leqq v \leqq \Theta$, and let $j \in\{-1,1\}$ and $0<K<\infty$ be such that $j b(u, v)<K$ for all $0<u<\delta$ and $0 \leqq v \leqq \Theta$. Now, for every $0<s<\delta$ and $0 \leqq \varepsilon \leqq \varepsilon_{0}$, define $G(s ; \varepsilon):=\varphi^{\prime}(s) / \theta(s ; \lambda, \varepsilon) \geqq 0$ and $H(s ; \varepsilon):=$ $\{K-j b(s,-\theta(s ; \lambda, 0))\} G(s ; \varepsilon) \geqq 0$. Applying the Mean Value Theorem, we can estimate

$$
H(s ; \varepsilon) \leqq K G(s ; \varepsilon)-j I(s, \theta(s ; \lambda, \varepsilon))+B(s)
$$

and

$$
|I(s, \theta(s ; \lambda, \varepsilon))| \leqq K G(s ; \varepsilon)+H(s ; \varepsilon)+B(s)
$$

for almost all $s \in(0, \delta)$ and all $0<\varepsilon \leqq \varepsilon_{0}$. Here $I(s, \theta(s ; \lambda, \varepsilon))$ is defined by (3.2). However, setting

$$
\beta:=K L+\int_{0}^{\delta} B(s) d s
$$

(4.11) yields

$$
\begin{aligned}
\int_{0}^{\delta} H(s ; \varepsilon) d s & \leqq \beta+\left|\int_{0}^{\delta} I(s ; \theta(s ; \lambda, \varepsilon)) d s\right| \\
& =\beta+|a(\delta,-\theta(\delta ; \lambda, \varepsilon))+\lambda \delta-a(0,-\varepsilon)| \\
& \leqq \beta+|a(\delta, 0)|+|a(\delta,-\Theta)|+|\lambda \delta|-a(0,-\varepsilon),
\end{aligned}
$$

for all $0<\varepsilon \leqq \varepsilon_{0}$, whence, in the limit $\varepsilon \downarrow 0$, the Monotone Convergence Theorem implies $H(s ; 0) \in L^{1}(0, \delta)$. Similarly, $(4.10)$ implies $G(s ; 0) \in L^{1}(0, \delta)$. It follows then from (4.12) that $|I(s, \theta(s ; \lambda, \varepsilon))| \leqq J(s)$ for almost all $s \in(0, \delta)$, where $J(s):=K G(s ; 0)+H(s ; 0)+B(s) \in L^{1}(0, \delta)$ for every $0<\varepsilon \leqq \varepsilon_{0}$. The Dominated Convergence Theorem subsequently infers

$$
I(s, \theta(s ; \lambda, 0))=\lim _{\varepsilon \downarrow 0} I(s, \theta(s ; \lambda, \varepsilon)) \in L^{1}(0, \delta),
$$

and one may take the limit $\varepsilon \downarrow 0$ in equation (3.1) for $\theta(s ; \lambda, \varepsilon)$ and in (4.10) to conclude that $\theta(s ; \lambda, 0)$ satisfies $(2.7)$ on $[0, \delta)$ and $(2.6)$. 
This completes the proof of the above assertion and therewith that of Lemma 3.3.

\section{Applications}

The 'singly nonlinear' forms of equations (1.1) and (1.2) are

$$
\frac{\partial u}{\partial t}=\frac{\partial}{\partial x}\left(\frac{\partial \varphi(u)}{\partial x}+\psi(u)\right)
$$

and

$$
\frac{\partial u}{\partial t}=\frac{\partial}{\partial x}\left(\frac{\partial \varphi(u)}{\partial x}+\psi(u)\right)+c(u)
$$

respectively, where, by analogy, $\varphi$ satisfies Hypothesis $1.1(\mathrm{i}), \psi \in C\left(\overline{\mathbb{R}^{+}}\right)$and $\psi(0)=$ 0 in equation (5.1); and $\varphi$ satisfies Hypothesis $1.2(\mathrm{i}), \psi \in C\left(\mathbb{R}^{+}\right) \cap C^{1}\left(\mathbb{R}^{+}\right), c \in C\left(\mathbb{R}^{+}\right)$, $c \varphi^{\prime} \in L^{1}(0, U)$ for all $U \in \mathbb{R}^{+}, \max \{0, c\} \in L^{\infty}(0, U)$ or $\max \{0,-c\} \in L^{\infty}(0, U)$ for all $U \in \mathbb{R}^{+}$, and $\psi(0)=c(0)=0$ in equation (5.2). Theorem 2.4 states that equation (5.1) admits finite speed of propagation if and only if there is a parameter $\lambda$ such that $\lambda s+\psi(s) \geqq 0$ for all $s \in[0, \delta)$ and

$$
\int_{0}^{\delta} \frac{\varphi^{\prime}(s)}{\lambda s+\psi(s)} d s<\infty \text { for some } 0<\delta<\infty,
$$

whilst equation (5.2) admits finite speed of propagation if and only if the integral equation

$$
\theta(s)=\lambda s+\psi(s)-\int_{0}^{s} \frac{c(r) \varphi^{\prime}(r)}{\theta(r)} d r
$$

has a non-negative solution $\theta$ on $[0, \delta)$ which satisfies (2.6). Noting that if there is a $\lambda^{*}$ such that $\lambda^{*} s+\psi(s) \geqq 0$ for all $s \in[0, \delta)$ then $\lambda s+\psi(s)>\lambda^{*} s+\psi(s) \geqq 0$ for all $s \in[0, \delta)$, the first result is equivalent to the statement that there is finite speed of propagation for (5.1) if and only if there is a $\lambda$ such that $\lambda s+\psi(s)>0$ for all $s \in[0, \delta)$ and (5.3) holds. This result was proved under stronger conditions on the functions $\varphi$ and $\psi$ in [27]. It was later generalised in $[29,30]$ to cover equation (5.1) under the conditions considered here, and to cover the result on equation (5.2) under even weaker conditions than those considered here. Prior to this, many results on finite speed of propagation of various examples of equations of the forms (5.1) and (5.2) were proved. We refer the reader to [30] for a historical survey of these and for a number of explicit results on the consequences for concrete examples of (5.1) and (5.2).

After the prototypical equation (1.3), the most-studied truly doubly nonlinear variant of equation (1.1) is probably

$$
\frac{\partial u}{\partial t}=\frac{\partial}{\partial x}\left(\Psi\left(\frac{\partial \varphi(u)}{\partial x}\right)\right)
$$

with $\varphi$ satisfying Hypothesis 1.1 (i) and with $\Psi \in C(\mathbb{R}), \Psi$ strictly increasing on $\mathbb{R}$, and $\Psi(0)=0[35,37,39,46,63,64]$. For this partial differential equation, the 
equation (2.5) becomes $\Psi(-\theta(s))+\lambda s=0$, which has a non-negative solution $\theta(s)=$ $-\Psi^{-1}(-\lambda s)$ which is not identically zero if and only if $\lambda>0$. Theorem 2.4 subsequently states that (5.4) displays finite speed of propagation if and only if there is a $\lambda>0$ such that

$$
\int_{0}^{\delta} \frac{\varphi^{\prime}(s)}{-\Psi^{-1}(-\lambda s)} d s<\infty \text { for some } 0<\delta<\infty .
$$

As a special case, it follows that (1.3) displays finite speed of propagation if and only if $m p>1$.

We note that the above also implies that equation (5.4) displays finite speed of propagation if $\Psi(v) v \leqq a_{0}|v|^{p+1}$ for all $v \in \mathbb{R}$ and $\varphi(u) \leqq \varphi_{0} u^{m}$ for all $u \in \mathbb{R}^{+}$for some real parameters $p>0, a_{0}, m>1 / p$ and $\varphi_{0}$, and that under these conditions with a few additional continuity requirements, given initial data with compact support, Yin [63] has proved the existence and uniqueness of a generalised solution with compact support for the Cauchy problem for equation (5.4).

With hindsight the sufficiency of (5.5) for finite speed of propagation of equation (5.4) was proved earlier in practically the same way as we have done above by Kalashnikov [35-38]. He also proved the necessity of (5.5) under extra conditions on $\varphi$ and $\Psi$ which he needed to complete the proof using explicitly constructed subsolutions in $[37,38]$. His work was marginally improved in [46]. Our method based on ideas in $[27,29,30]$, is different, and uses only travelling waves as it were. This approach has also been followed in [56]. This approach leads to the avoidance of the extra conditions.

A generalisation of the above result is the following theorem:

THEOREM 5.1. The equation

$$
\frac{\partial u}{\partial t}=\frac{\partial}{\partial x}\left(D(u) \Psi\left(\frac{\partial \varphi(u)}{\partial x}\right)\right),
$$

with $\varphi$ satisfying Hypothesis $1.1(\mathrm{i})$, with $\Psi \in C(\mathbb{R}), \Psi$ strictly increasing on $\mathbb{R}$ and $\Psi(0)=0$, and with $D \in C\left(\overline{\mathbb{R}^{+}}\right)$and $D(u)>0$ for all $u \geqq 0$, admits finite speed of propagation if and only if there is a $\lambda>0$ such that (5.5) holds.

Proof. Taking $a(u, v)=D(u) \Psi(v)$, the equation (2.5) becomes simply $D(s) \Psi(-\theta(s))+\lambda s=0$. For all $\lambda \geqq 0$, this admits the non-negative solution $\theta(s)=$ $-\Psi^{-1}(-\lambda s / D(s))$ on some interval $[0, \delta(\lambda))$. Moreover, by the continuity of $D$ and the monotonicity of $\Psi$, we can estimate $-\Psi^{-1}(-\lambda s / 2 D(0)) \leqq \theta(s) \leqq$ $-\Psi^{-1}(-2 \lambda s / D(0))$ for all sufficiently small $s \in[0, \delta(\lambda))$. Hence, if we consider $\lambda$ in (5.5) as an arbitrary generic positive constant not necessarily identified with that in equation (2.5), there is a solution $\theta$ of (2.5) which satisfies (2.6) if and only if there is a $\lambda>0$ such that $(5.5)$ holds.

We remark that in general it is not possible to remove the dependence on $\lambda$ from the statement of Theorem 5.1. By way of a counter-example, consider equation (5.4) with

$$
\Psi(v)= \begin{cases}\Psi_{0} v /|v \ln | v|| & \text { for }|v| \leqq 1 / 2 \\ 2 \Psi_{0} v /(\ln 2) & \text { for }|v|>1 / 2\end{cases}
$$


for some $\Psi_{0}>0$, and with $\varphi(u)=u^{m} \exp (-1 / u)$ for some $m>0$. In this case the integral condition (5.5) is equivalent to

$$
\int_{0}^{\delta}(1+m s) s^{m-2} \exp \left(\left(\Psi_{0}-\lambda\right) / \lambda s\right) d s<\infty \quad \text { for some } 0<\delta<\Psi_{0} /(\lambda \ln 2) .
$$

It can be seen that this criterion is negated for any $\lambda<\Psi_{0}$, whilst it is satisfied for all $\lambda>\Psi_{0}$. Furthermore, for the marginal parameter value $\lambda=\Psi_{0}$ the condition is satisfied when, but only when, $m>1$.

A detailed study of the Cauchy problem for a particularly pathological example of equation $(5.6)$ can be found in $[\mathbf{1 0}, 11,18]$.

A further example of equation (1.1) which has received attention $[7,17]$ in the past is the equation

$$
\frac{\partial u}{\partial t}=\frac{\partial}{\partial x}\left(D(u)\left|\frac{\partial u}{\partial x}\right|^{p-1} \frac{\partial u}{\partial x}\right)
$$

where $D \in C\left(\overline{\mathbb{R}^{+}}\right), D(u)>0$ for almost all $u>0$, and $p>0$. This equation may be incorporated in our theory by formulating it as an equation of the type (1.1) with

$$
\varphi(u)=\int_{0}^{u} D^{1 / p}(s) d s \quad \text { and } \quad a(u, v)=|v|^{p-1} v .
$$

In this case equation (2.5) takes the form $-|\theta(s)|^{p-1} \theta(s)+\lambda s=0$. This admits a nonnegative solution $\theta(s)=(\lambda s)^{1 / p}$ if and only if $\lambda \geqq 0$ and consequently one which satisfies (2.6) if and only if $\lambda>0$ and $\varphi^{\prime}(s) / s^{1 / p} \in L^{1}(0, \delta)$ for some $0<\delta<\infty$, where $\varphi$ is given by (5.8). The outcome is thus the following theorem:

THEOREM 5.2. Equation (5.7), with $D \in C\left(\overline{\mathbb{R}^{+}}\right), D(u)>0$ for almost all $u>0$, and $p>0$, admits finite speed of propagation if and only if

$$
\int_{0}^{\delta} \frac{D^{1 / p}(s)}{s^{1 / p}} d s<\infty \quad \text { for some } 0<\delta<\infty .
$$

Previously Atkinson and Bouillet [7] had shown that the above condition was necessary and sufficient for (5.7) to admit a particular type of similarity solution with compact support, and in [17] discussed the consequences of this result for arbitrary solutions of (5.7). Taking $D(u)=m^{p} u^{p(m-1)}$ in Theorem 5.2 again gives the criterion that (1.3) has finite speed of propagation if and only if $m p>1$.

An alternative generalisation of the results for equation (5.4) may be obtained for the archetype of the equation studied by Díaz and de Thelin [19], i.e.

$$
\frac{\partial u}{\partial t}=\frac{\partial}{\partial x}\left(\left|\frac{\partial \varphi(u)}{\partial x}+\psi(u)\right|^{p-1}\left(\frac{\partial \varphi(u)}{\partial x}+\psi(u)\right)\right) .
$$

THEOREM 5.3. Let $p>0$, let $\varphi$ satisfy Hypothesis $1.1(\mathrm{i})$, and let $\psi \in C\left(\overline{\mathbb{R}^{+}}\right)$with $\psi(0)=0$. Then equation (5.9) displays finite speed of propagation if and only if

$$
\max \{0,-\psi(s)\}=\mathcal{O}\left(s^{1 / p}\right) \quad \text { as } s \downarrow 0
$$


and

$$
\int_{0}^{\delta} \frac{\varphi^{\prime}(s)}{\max \left\{\psi(s), s^{1 / p}\right\}} d s<\infty \quad \text { for some } 0<\delta<\infty .
$$

Proof. Equation (5.9) is an equation of the type (1.1) with $a(u, v)=$ $|\psi(u)+v|^{p-1}(\psi(u)+v)$. Subsequently the equation (2.5) becomes $|\psi(s)-\theta(s)|^{p-1}(\psi(s)-\theta(s))+\lambda s=0$, which has as the only admissible solution

$$
\theta(s)=\psi(s)+\lambda|\lambda|^{(1-p) / p} s^{1 / p}
$$

It follows that (2.5) has a non-negative solution $\theta$ on some interval $[0, \delta)$ only if $\lambda|\lambda|^{(1-p) / p} s^{1 / p} \geqq-\psi(s)$ for all $s \in[0, \delta)$. This yields the necessity of $(5.10)$. On the other hand, if (5.10) holds and one defines

$$
\sigma:=\limsup _{s \downarrow 0} s^{-1 / p} \max \{0,-\psi(s)\},
$$

and $\delta:=\sup \left\{s>0:(\sigma+1)>-r^{1 / p} \psi(r)\right.$ for all $\left.r \in(0, s)\right\}$, then for all $\lambda \geqq(\sigma+2)^{p}$ and $s \in(0, \delta)$ one has

$$
\begin{aligned}
\left(1+\lambda^{1 / p}\right) \max \left\{\psi(s), s^{1 / p}\right\} & \geqq \psi(s)+|\lambda s|^{1 / p} \\
& =(\sigma+1) s^{1 / p}+\psi(s)+|\lambda s|^{1 / p}-(\sigma+1) s^{1 / p} \\
& \geqq \max \left\{(\sigma+1) s^{1 / p}+\psi(s),|\lambda s|^{1 / p}\right\}-(\sigma+1) s^{1 / p} \\
& =\max \left\{\psi(s),\left(|\lambda|^{1 / p}-\sigma-1\right) s^{1 / p}\right\} \\
& \geqq \max \left\{\psi(s), s^{1 / p}\right\} .
\end{aligned}
$$

Subsequently the function given by (5.12) satisfies (2.6) for large $\lambda$ if and only if (5.11) holds.

COROllary 5.4. Consider the equation

$$
\frac{\partial u}{\partial t}=\frac{\partial}{\partial x}\left(\left|\frac{\partial u^{m}}{\partial x}+\psi_{0} u^{n}\right|^{p-1}\left(\frac{\partial u^{m}}{\partial x}+\psi_{0} u^{n}\right)\right)
$$

with $m>0, n>0, p>0$ and $\psi_{0}$ real parameters.

(i) If $\psi_{0}<0$ then this equation admits finite speed of propagation if and only if $m p>1$ and $n p \geqq 1$.

(ii) If $\psi_{0}>0$ then this equation admits finite speed of propagation if and only if $m p>1$ or $m>n$.

Note that the travelling-wave solutions and subsolutions which we essentially use to prove our main theorem and thereby Theorem 5.3 are such that their partial derivatives with respect to $t$ are locally integrable, which condition occurs in the comparison principle for equation (5.9) proved by Díaz and de Thelin [19].

The reader may like to compare the last result with the following, whose proof is similar and therefore omitted.

THEOREM 5.5. The equation

$$
\frac{\partial u}{\partial t}=\frac{\partial}{\partial x}\left(\left|\frac{\partial \varphi(u)}{\partial x}\right|^{p-1} \frac{\partial \varphi(u)}{\partial x}+\psi(u)\right)
$$


where $p>0$, $\varphi$ satisfies Hypothesis $1.1(\mathrm{i})$ and $\psi \in C\left(\overline{\mathbb{R}^{+}}\right)$with $\psi(0)=0$, admits finite speed of propagation if and only if

$$
\max \{0,-\psi(s)\}=\mathcal{O}(s) \quad \text { as } s \downarrow 0
$$

and

$$
\int_{0}^{\delta} \frac{\varphi^{\prime}(s)}{\max \{\psi(s), s\}^{1 / p}} d s<\infty \quad \text { for some } 0<\delta<\infty .
$$

A peculiar example of equation (1.1) which arises in the study of hydrology is the equation

$$
\frac{\partial u}{\partial t}=\frac{\partial}{\partial x}\left(D(u) \frac{\partial u}{\partial x} /\left\{1+\left(\frac{\partial u}{\partial x}\right)^{2}\right\}\right),
$$

where $D \in C^{1}([0,1])$ with $D(0)=D(1)=0$ and $D^{\prime}(0)>0>D^{\prime}(1)$ and $D(u)>0$ for all $u \in(0,1)$. In this model, finite speed of propagation is associated with an interface denoting a front of salt-water penetration in a freshwater aquifier [32]. This equation has been studied in some detail by van Duijn and others $[12,13,22-24]$. At first glance, this equation falls out of the scope of our analysis, since the function $a(u, v)=$ $D(u) v /\left(1+v^{2}\right)$ does not satisfy the monotonicity requirement in Hypothesis 1.1(ii). Nonetheless, we observe that the previously mentioned authors consider only solutions for which $0 \leqq u \leqq 1$ and for which the generalised derivative $|\partial u / \partial x| \leqq 1$ almost everywhere. Hence, if we define

$$
\varphi(u)= \begin{cases}\int_{0}^{u} D(s) d s & \text { for } u \leqq 1, \\ u \int_{0}^{1} D(s) d s & \text { for } u>1,\end{cases}
$$

and

$$
a(u, v)= \begin{cases}\left\{D(u)+(v-D(u))^{2}\right\} / 2 & \text { for } v>D(u), \\ D^{2}(u) v /\left\{D^{2}(u)+v^{2}\right\} & \text { for }|v| \leqq D(u), \\ -\left\{D(u)+(v+D(u))^{2}\right\} / 2 & \text { for } v<-D(u),\end{cases}
$$

we have an equivalent equation of the class (1.1) which does fit into our analysis. With $\varphi$ and $a$ given by (5.14) and (5.15), respectively, for any $0<\lambda<D^{\prime}(0) / 2$, it can be checked that the equation (2.5) admits the explicit solution

$$
\theta(s)=2 \lambda s D(s) /\left\{D(s)+\sqrt{D^{2}(s)-4 \lambda^{2} s^{2}}\right\} .
$$

Noting that for this solution there holds

$$
\varphi^{\prime}(s) / \theta(s) \rightarrow\left\{D^{\prime}(0)+\sqrt{\left(D^{\prime}(0)\right)^{2}-4 \lambda^{2}}\right\} / 2 \lambda \quad \text { as } s \downarrow 0,
$$

it follows that in this case one can explicitly check that (2.5) has a solution satisfying (2.6) for every $0<\lambda<D^{\prime}(0) / 2$. Whence, according to our theory, equation (5.13) displays finite speed of propagation, as was proved earlier by van Duijn and Zhang [22].

In the past, sufficient conditions for finite speed of propagation by equations of 
the types (1.1) and (1.2) have been determined successfully by a technique known as the energy method $[\mathbf{2 - 6 , 2 0}$. To demonstrate the relationship between those results and our criteria, we consider the following theorem:

THEOREM 5.6. Suppose that $\varphi$ satisfies Hypothesis 1.1(i), a satisfies Hypothesis 1.1(ii), and $b \in C\left(\mathbb{R}^{+} \times \mathbb{R}\right)$ with $b(0,0)=0$. Suppose, furthermore, that there exists an integer $N \geqq 1$ and real parameters $m>0, \varphi_{0}>0, p>0, a_{1}>a_{0}>0, \alpha_{i}$ and $\beta_{i}$ for $1 \leqq i \leqq N$, and $B \geqq 0$ such that

$$
\begin{gathered}
\varphi(u) \leqq \varphi_{0} u^{m}, \\
a_{0}|v|^{p+1} \leqq a(u, v) v \leqq a_{1}|v|^{p+1}
\end{gathered}
$$

and

$$
|b(u, v)| \leqq B \sum_{i=1}^{N} \varphi^{\alpha_{i}}(u)|v|^{\beta i}
$$

for all $0<u<U$ and $0<|v|<U$, some $U<\infty$. Then equation (1.2) displays finite speed of propagation whenever

$$
m p>1 \text {, }
$$

and

$$
\alpha_{i}>-1 \text { and } \beta_{i} \geqq p+1-m p\left(\alpha_{i}+1\right) \quad \text { for each } 1 \leqq i \leqq N .
$$

Theorems based on the energy method similar to this may be found in the references $[2-6,20]$. However, besides also applying to nonautonomous equations and to equations in an arbitrary number of space dimensions, those theorems do not require any monotonicity assumptions on $\varphi$ and $a$. Notwithstanding this, except on two counts, under the stated hypotheses Theorem 5.6 improves on the aforementioned results. The first count is that finite speed of propagation has also been proved using the energy method with the added restriction.

$$
B<a_{0}
$$

in the marginal case

$$
\alpha_{1}=-1, \quad \beta_{1}=p+1 \quad \text { and } N=1
$$

by Díaz and Veron [20] (see also [4]). The second count is that applying the energy method with $\varphi, a$ and $b$ satisfying the conditions of the form (5.16)-(5.20) or $(5.16)-(5.19),(5.21),(5.22)$ in $[\mathbf{5 , 6 , 2 0}]$, the analysis extends to

$$
\frac{\partial u}{\partial t}=\frac{\partial}{\partial x}\left(a\left(u, \frac{\partial \varphi(u)}{\partial x}\right)\right)+b\left(u, \frac{\partial \varphi(u)}{\partial x}\right)+c(u),
$$

where $c(u) \leqq 0$ for all $u \geqq 0$. We note, though, that this is also a trivial extension in our situation if we replace a weak solution $v$ in our comparison principle Hypothesis 2.3(i) by a weak supersolution $v$.

Proof of Theorem 5.6. For fixed $\lambda>0$, consider the mapping (4.7) with $s^{*}=0$ and $\rho=0$ defined on the set of functions $S:=\{\theta \in C([0, \delta]):-2 \lambda s \leqq a(s,-\theta(s)) \leqq-\lambda s / 2$ for all $s \in[0, \delta]\}$ for some $0<\delta<U$. It can be shown that if (5.16)-(5.19) hold and if $\alpha_{i}>-1$ and $\beta_{i}>p+1-m p\left(\alpha_{i}+1\right)$ for each $1 \leqq i \leqq N$, then (4.7) with $s^{*}=0$ and 
$\rho=0$ maps $S$ into itself for sufficiently small $\delta$. Under conditions (5.16)-(5.19) and (5.20), the same can be said for all sufficiently large $\lambda$. Subsequently, for appropriate $\lambda>0$ and $0<\delta<U$, we can construct a Peano sequence on $S$ by defining $\theta_{0}(s):=-A(s,-\lambda s)$ and

$$
\theta_{k}(s):=-A\left(s,-\lambda s+\int_{0}^{s} \frac{b\left(r,-\theta_{k-1}(r)\right) \varphi^{\prime}(r)}{\theta_{k-1}(r)} d r\right)
$$

for every $k \geqq 1$, where $A$ is given by (4.4). This sequence of functions is equicontinuous and hence contains a subsequence which converges to a solution $\theta$ of (2.7) in $S$. Moreover, we can estimate

$$
\begin{aligned}
\int_{0}^{\delta} \frac{\varphi^{\prime}(s)}{\theta(s)} d s & \leqq\left(\frac{2 a_{1}}{\lambda}\right)^{1 / p} \int_{0}^{\delta} \frac{\varphi^{\prime}(s)}{s^{1 / p}} d s \\
& \leqq\left(\frac{2 a_{1} \varphi_{0}^{1 / m}}{\lambda}\right)^{1 / p} \int_{0}^{\delta} \frac{\varphi^{\prime}(s)}{\varphi^{1 / m p}(s)} d s \\
& =\frac{m p}{m p-1}\left(\frac{2 a_{1} \varphi_{0}^{1 / m}}{\lambda}\right)^{1 / p} \varphi^{(m p-1) / m p}(\delta)<\infty,
\end{aligned}
$$

when (5.19) holds. It follows that for sufficiently large $\lambda$, equation (2.7) has a solution which satisfies (2.6), whence, by Corollary 3.4 to Theorem 2.4, equation (1.2) displays finite speed of propagation.

For a very specific doubly nonlinear degenerate parabolic equation, Nagai [47] has shown that the Cauchy problem with compactly supported initial data admits a solution with compact support. His work implies that if $p \geqq 1, \varphi(u) \equiv u$, $c \in C\left(\mathbb{R}^{+}\right) \cap L^{1}(0, U)$ for all $U \in \mathbb{R}^{+}, c(u) \leqq-\gamma$ for some constant $\gamma>0$ for all $u \in \mathbb{R}^{+}$, and $c(0)=0$, then the equation

$$
\frac{\partial u}{\partial t}=\frac{\partial}{\partial x}\left(\left|\frac{\partial \varphi(u)}{\partial x}\right|^{p-1} \frac{\partial \varphi(u)}{\partial x}\right)+c(u)
$$

displays finite speed of propagation. The next result captures this conclusion.

THEOREM 5.7. Suppose that $p>0, \varphi$ satisfies Hypothesis $1.2(\mathrm{i}), \quad c \in C\left(\mathbb{R}^{+}\right)$, $c \varphi^{\prime} \in L^{1}(0, U)$ for all $U \in \mathbb{R}^{+}$, and $c(0)=0$. Set

$$
G(s):=\int_{0}^{s}|c(r)| \varphi^{\prime}(r) d r
$$

(a) When $c(u) \leqq 0$ for all $u \in \mathbb{R}^{+}$, equation (5.23) displays finite speed of propagation if and only if

$$
\int_{0}^{\delta} \frac{\varphi^{\prime}(s)}{\max \left\{s^{1 / p}, G^{1 /(p+1)}(s)\right\}} d s<\infty \quad \text { for some } 0<\delta<\infty .
$$

(b) When $c(u) \geqq 0$ for all $u \in \mathbb{R}^{+}$, equation (5.23) displays finite speed of propagation if and only if

$$
G(s)=\mathcal{O}\left(s^{(p+1) / p}\right) \quad \text { as } s \downarrow 0
$$


and

$$
\int_{0}^{\delta} \frac{\varphi^{\prime}(s)}{s^{1 / p}} d s<\infty \quad \text { for some } 0<\delta<\infty .
$$

Proof. (a) In this particular situation, equation (2.7) reads as

$$
\theta^{p}(s)=\lambda s+\int_{0}^{s} \frac{G^{\prime}(r)}{\theta(r)} d r
$$

for non-negative solutions. Moreover, it can be checked that

$$
\theta_{0}(s):=\left(\frac{p+1}{p} G(s)\right)^{1 /(p+1)}
$$

is a solution of this integral equation when $\lambda=0$. Subsequently, by Proposition 4.5 and (4.2), for any $\lambda \geqq 0$ equation (5.26) has a maximal solution $\theta(s ; \lambda, 0)$ on an interval $[0, M(\lambda, 0))$ such that

$$
\theta(s ; \lambda, 0) \geqq \theta_{0}(s) \text { for all } s \in[0, M(\lambda, 0)) .
$$

Plainly from (5.26) there holds $\theta^{p}(s ; \lambda, 0) \geqq \lambda s$ for all $s \in[0, M(\lambda, 0))$, and substituting (5.27) in the right-hand side of (5.26) there holds $\theta^{p}(s ; \lambda, 0) \leqq \lambda s+\theta_{0}^{p}(s)$ for all $s \in[0, M(\lambda, 0))$ and $\lambda \geqq 0$. It follows that for every $\lambda \geqq 0$ necessarily $M(\lambda, 0)=\infty$ and

$$
\begin{gathered}
\min \left\{\lambda^{1 / p},\left(\frac{p+1}{p}\right)^{1 /(p+1)}\right\} \max \left\{s^{1 / p}, G^{1 /(p+1)}(s)\right\} \leqq \theta(s ; \lambda, 0) \\
\left.\quad \leqq \lambda+\left(\frac{p+1}{p}\right)^{p /(p+1)}\right\}^{1 / p} \max \left\{s^{1 / p}, G^{1 /(p+1)}(s)\right\}
\end{gathered}
$$

for all $s \in[0, \infty)$. The theorem now follows by testing $\theta(s ; \lambda, 0)$ for $\lambda>0$ in the criterion (2.6).

(b) In this case equation (2.7) reads as

$$
\theta^{p}(s)=\lambda s-\int_{0}^{s} \frac{G^{\prime}(r)}{\theta(r)} d r
$$

for non-negative solutions. Clearly then, if there is to be a non-negative solution satisfying (2.6), necessarily $\lambda>0$. Suppose now that (5.28) does have a non-negative solution $\theta$ in the sense of Definition 3.1 on an interval $[0, \delta)$ for some $\lambda>0$. Then, setting

$$
Y(s):=\int_{0}^{s} \frac{G^{\prime}(r)}{\theta(r)} d r
$$

with the integrand defined on the lines of (3.2), it can be verified that $G^{\prime}(s)=$ $\theta(s) Y^{\prime}(s)=(\lambda s-Y(s))^{1 / p} Y^{\prime}(s)$ for almost all $s \in(0, \delta)$. Consequently,

$$
\begin{aligned}
G(s) & =\int_{0}^{s}(\lambda r-Y(r))^{1 / p} Y^{\prime}(r) d r \\
& \leqq \int_{0}^{s}(\lambda s-Y(r))^{1 / p} Y^{\prime}(r) d r
\end{aligned}
$$




$$
=\frac{p}{p+1}\left\{(\lambda s)^{(p+1) / p}-(\lambda s-Y(s))^{(p+1) / p}\right\},
$$

for all $s \in(0, \delta)$. This yields (5.24). Suppose, on the other hand, that (5.24) holds. Let $\gamma>0$ and $\delta>0$ be such that $G(s) \leqq \gamma s^{(p+1) / p}$ for all $s \in(0, \delta)$. Set

$$
\theta_{0}(s):=s^{1 / p}
$$

and for all $k \geqq 1$ define

$$
\theta_{k}(s):=\left(\lambda s-\int_{0}^{s} \frac{G^{\prime}(r)}{\theta_{k-1}(r)} d r\right)^{1 / p} .
$$

If $\theta_{k-1}(s) \geqq s^{1 / p}$ on $[0, \delta)$, we can estimate

$$
\begin{aligned}
\theta_{k}^{p}(s) & \geqq \lambda s-\int_{0}^{s} \frac{G^{\prime}(r)}{r^{1 / p}} d r \\
& \geqq \lambda s-\gamma^{1 /(p+1)} \int_{0}^{s} \frac{G^{\prime}(r)}{G^{1 /(p+1)}(r)} d r \\
& =\lambda s-\frac{p+1}{p} \gamma^{1 /(p+1)} G^{p /(p+1)}(s) \\
& \geqq \lambda s-\frac{p+1}{p} \gamma s,
\end{aligned}
$$

for all $s \in(0, \delta)$. It follows that if $\lambda>1+(p+1) \gamma / p$, then (5.29), (5.30) defines a nondecreasing sequence of functions $\left\{\theta_{k}\right\}_{k=0}^{\infty}$ on $[0, \delta)$ such that $s^{1 / p} \leqq \theta_{k}(s) \leqq(\lambda s)^{1 / p}$ for all $s \in[0, \delta)$ and $k \geqq 0$. Hence this sequence has a pointwise limit which by (5.30) must be a solution of (5.28). We conclude that for all sufficiently large $\lambda$, equation (5.28) has a maximal solution $\theta(s ; \lambda, 0)$ on $[0, \delta)$ such that $\theta(s ; \lambda, 0) \geqq s^{1 / p}$ for all $s \in[0, \delta)$. Since, though, by (5.28) necessarily $\theta(s ; \lambda, 0) \leqq(\lambda s)^{1 / p}$ for all $s \in[0, \delta)$, this means that the criterion (2.6) is equivalent to (5.25).

For more general forms of equation (1.2) the most complete results on finite speed of propagation obtained to date are those of $\mathrm{Su}[\mathbf{5 5}, \mathbf{5 6}]$. He considered the particular form

$$
\frac{\partial u}{\partial t}=\frac{\partial}{\partial x}\left(\Psi\left(\frac{\partial \varphi(u)}{\partial x}\right)+\psi(u)\right)+c(u),
$$

where $\varphi \in C\left(\overline{\mathbb{R}^{+}}\right) \cap C^{1}\left(\mathbb{R}^{+}\right), \quad \varphi^{\prime}(u)>0$ for all $u \in \mathbb{R}^{+}$and $\varphi(0)=0$, where $\Psi \in C(\mathbb{R}) \cap C^{1}\left(\mathbb{R}^{+}\right) \cap C^{1}\left(\mathbb{R}^{-}\right), \Psi^{\prime}(v)>0$ for all $v \in \mathbb{R}^{+} \cup \mathbb{R}^{-}, \Psi(v) \rightarrow \pm \infty$ as $v \rightarrow \pm \infty$ and $\Psi(0)=0$, where $\psi \in C\left(\mathbb{R}^{+}\right)$and $\psi(0)=0$, and finally where $c \in C\left(\overline{\mathbb{R}^{+}}\right) \cap C^{1}\left(\mathbb{R}^{+}\right), c(u) \leqq 0$ for all $u \in \overline{\mathbb{R}^{+}}$and $c(0)=0$. The results he obtained can be shown to be equivalent to ours.

To be specific, $\mathrm{Su}$ [56] shows that if there exists a $\lambda \in \mathbb{R}$ and a function $f \in C([0, \infty)) \cap C^{1}(0, \infty)$ such that $f(0)=0, f^{\prime}(z)>0$ and

$$
(\Psi(-f))^{\prime}(z) \geqq-\lambda\left(\varphi^{-1}\right)^{\prime}(z)-\left(\psi\left(\varphi^{-1}\right)\right)^{\prime}(z)+c\left(\varphi^{-1}(z)\right) / f(z)
$$


for all $z \in(0, \delta)$, and

$$
\int_{0}^{\delta} \frac{d z}{f(z)}<\infty \text { for some } 0<\delta<\infty,
$$

then equation (5.31) displays finite speed of propagation. Whereas, if, for every $\lambda \in \mathbb{R}$, there is a function $f \in C([0, \infty)) \cap C^{1}(0, \infty)$ such that $f(0)=0, f^{\prime}(z)>0$ and

$$
(\Psi(-f))^{\prime}(z) \leqq-\lambda\left(\varphi^{-1}\right)^{\prime}(z)-\left(\psi\left(\varphi^{-1}\right)\right)^{\prime}(z)+c\left(\varphi^{-1}(z)\right) / f(z)
$$

for all $z \in(0, \delta)$, and

$$
\int_{0}^{\delta} \frac{d z}{f(z)}=\infty \text { for some } 0<\delta<\infty,
$$

then the equation does not display finite speed of propagation. Noting that, adapting techniques in [28], under Su's conditions for finite speed of propagation it can be shown that equation (2.7) has a unique solution $\theta$ such that $\theta(s) \geqq f(\varphi(s))$ for all $s \in[0, \delta)$, whilst under Su's conditions for the absence of finite speed of propagation it can be shown that this integral equation either has no solution or a unique solution $\theta$ on an interval $[0, M)$ such that $\theta(s) \leqq f(\varphi(s))$ for all $s \in[0, \min \{M, \delta\})$ for the respective function $f$, Su's results are indeed covered by ours.

As a special case of equation (5.31), consider the equation

$$
\frac{\partial u}{\partial t}=\frac{\partial}{\partial x}\left(\left|\frac{\partial u^{m}}{\partial x}\right|^{p-1} \frac{\partial u^{m}}{\partial x}+\psi_{0} u^{n}\right)+c(u),
$$

with

$$
c(u)= \begin{cases}c_{0} u^{q} & \text { if } u>0 \\ 0 & \text { if } u=0\end{cases}
$$

in which

$$
m>0, \quad p>0, \quad \psi_{0}, \quad n>0, \quad c_{0} \text { and } q>-m
$$

are real parameters. Conditions for the equation with $c_{0} \leqq 0$ and $q>0$ to admit a travelling-wave solution with an interface of the type (2.4) were determined by Pavlov, Pokrovskii and Taranenko [49]. In the theorem below, we present the necessary and sufficient conditions for finite speed of propagation for this equation for the parameter range indicated, as a consequence of Theorem 2.4. The results for the case that $c_{0} \leqq 0$ and $q>0$ are identical to those obtained previously by $\mathrm{Su}$ $[55,56]$. We note that, for the more general case, finite speed of propagation has also previously been investigated using formal methods by Pokrovskii and Taranenko $[\mathbf{5 0}]$.

THEOREM 5.8. Equation (5.32) (5.34) displays finite speed of propagation if and only if one of the following hold.

(i) $c_{0}<0, n \geqq 1$ or $\psi_{0}=0$, and $m p>\min \{q, 1\}$.

(ii) $c_{0}<n, n<1, \psi_{0}<0$ and $\min \{m p, n\}>q$.

(iii) $c_{0}<0, n<1, \psi_{0}>0$ and $m p>\min \{q, n\}$.

(iv) $c_{0}=0, n \geqq 1$ or $\psi_{0}=0$, and $m p>1$.

(v) $c_{0}=0, n<1, \psi_{0}>0$ and $m p>n$. 
(vi) $c_{0}>0, n \geqq 1$ or $\psi_{0}=0, m p>1$ and $(m+q) p \geqq p+1$.

(vii) $c_{0}>0, n<1,0<\psi_{0}<(p+1)\left(m c_{0} / n p\right)^{p /(p+1)}, m p>n$ and $(m+q) p>(p+1) n$.

(viii) $c_{0}>0, n<1, \psi_{0} \geqq(p+1)\left(m c_{0} / n p\right)^{p /(p+1)}, m p>n$ and $(m+q) p \geqq(p+1) n$.

Proof. For the particular case of equations (5.32)-(5.34), the integral equation (2.7) becomes

$$
-|\theta(s)|^{p-1} \theta(s)+\psi_{0} s^{n}+\lambda s=\int_{0}^{s} \frac{m c_{0} r^{m+q-1}}{\theta(r)} d r .
$$

Setting $f(s):=\lambda s+\psi_{0} s^{n}, \quad g(s):=-m c_{0} s^{m+q-1}$ and $\Theta(s)=\theta^{p}(s)$ for non-negative solutions, this equation is equivalent to

$$
\Theta(s)=f(s)+\int_{0}^{s} \frac{g(r)}{\Theta^{1 / p}(r)} d r .
$$

This last equation yields an equation of the class (4.6) in the special case $p=1$. By extending the analysis for (4.6) in [28] to (5.36), it can be shown that (5.35) has a continuous non-negative solution in a right neighbourhood of zero if and only if the parameters in the equation satisfy one of the following eight combinations. Moreover, in each case, by choosing $\lambda$ large enough, the maximal solution $\theta(s ; \lambda, 0)$ must satisfy

$$
\theta(s ; \lambda, 0) \sim \theta_{0} s^{\alpha} \quad \text { as } s \downarrow 0,
$$

with the value of $\alpha$ shown for some $\theta_{0}>0$ which may depend on $\lambda$.

(i) $c_{0}<0, n \geqq 1$ or $\psi_{0}=0$; with $\alpha=\min \{(m+q) /(p+1), 1 / p\}$;

(ii) $c_{0}<0, n<1, \psi_{0}<0$; with $\alpha=\max \{(m+q) /(p+1), m+q-n\}$;

(iii) $c_{0}<0, n<1, \psi_{0}>0$; with $\alpha=\min \{(m+q) /(p+1), n / p\}$;

(iv) $c_{0}=0, n \geqq 1$ or $\psi_{0}=0$; with $\alpha=1 / p$;

(v) $c_{0}=0, n<1, \psi_{0}>0$; with $\alpha=n / p$;

(vi) $c_{0}>0, n \geqq 1$ or $\psi_{0}=0,(m+q) p \geqq p+1$; with $\alpha=1 / p$;

(vii) $c_{0}>0, n<1,0<\psi_{0}<(p+1)\left(m c_{0} / n p\right)^{p /(p+1)},(m+q) p>(p+1) n$; with $\alpha=$ $n / p$

(viii) $c_{0}>0, n<1, \psi_{0} \geqq(p+1)\left(m c_{0} / n p\right)^{p / p+1},(m+q) p \geqq(p+1) n$; with $\alpha=n / p$.

The theorem now follows by testing (2.6) via the equivalent criterion

$$
\int_{0}^{\delta} \frac{m s^{m-1}}{\theta_{0} s^{\alpha}} d s<\infty \text { for some } 0<\delta<\infty .
$$

\section{Acknowledgment}

This work was supported in part by Research Fund OTKA T-4374 of the Hungarian Academy of Sciences.

\section{References}

1 H. W. Alt and S. Luckhaus. Quasilinear elliptic-parabolic differential equations. Math. Z. 183 (1983), 311-41.

2 S. N. Antoncev. The character of perturbations described by solutions of multidimensional degenerate parabolic equations. Dinamika Sploshn. Sredy 40 (1979), 114-22 (in Russian).

$3 \mathrm{~S}$. N. Antoncev. On the localization of solutions of nonlinear degenerate elliptic and parabolic 
equations. Soviet Math. Dokl. 24 (1981), 420-24; originally appearing in Russian as: Dokl. Akad. Nauk SSSR 260 (1981), 1289-93.

4 S. N. Antontsev. Metastable localization of solutions of degenerate parabolic equations of general form. Dinamika Sploshn. Sredy 83 (1987), 138-44 (in Russian).

5 S. N. Antontsev and Kh. I. Dias. New results on localization of solutions of nonlinear elliptic and parabolic equations obtained by the energy method. Soviet Math. Dokl. 38 (1989), 535-9; originally appearing in Russian as: Dokl. Akad. Nauk SSSR 303 (1988), 524-9.

6 S. N. Antontsev and S. I. Shmarëv. The local energy method and vanishing of weak solutions of nonlinear parabolic equations. Soviet Math. Dokl. 43 (1991), 738-42; originally appearing in Russian as: Dokl. Akad. Nauk SSSR 318 (1991), 777-81.

7 C. Atkinson and J. E. Bouillet. Some qualitative properties of solutions of a generalised diffusion equation. Math. Proc. Cambridge Philos. Soc. 86 (1979), 495-510.

8 A. Bamberger. Étude d'une équation doublement non linéaire. J. Funct. Anal. 24 (1977), 148-55.

9 G. I. Barenblatt. On self-similar motions of a compressible fluid in a porous medium. Prikl. Mat. Mekh. 16 (1952), 679-98 (in Russian).

10 M. Bertsch and R. Dal Passo. Hyperbolic phenomena in a strongly degenerate parabolic equation. Arch. Rational Mech. Anal. 117 (1992), 349-87.

$11 M$. Bertsch and R. Dal Passo. A parabolic equation with a mean-curvature type operator. In Nonlinear Diffusion Equations and Their Equilibrium States 3, eds N. G. Lloyd, W. M. Ni, L. A. Peletier and J. Serrin, 89-97 (Boston: Birkhäuser, 1992).

12 M. Bertsch, C. J. van Duijn, J. R. Esteban and H. Zhang. Regularity of the free boundary in a doubly degenerate parabolic equation. Comm. Partial Differential Equations 14 (1989), 391-412.

13 M. Bertsch, J. R. Esteban and H. Zhang. On the asymptotic behaviour of the solutions of a degenerate equation in hydrology. Nonlinear Anal. 19 (1992), 365-74.

14 P. Blanc. A degenerate parabolic equation. In Semigroup Theory and Applications, eds P. Clément, S. Invernizzi, E. Mitidieri and I. I. Vrabie, 59-65 (New York: Marcel Dekker, 1989).

15 P. Blanc. Existence de solutions discontinues pour des équations paraboliques. C. R. Acad. Sci. Paris Sér. I Math. 310 (1990), 53-6.

16 P. Blanc. On the regularity of the solutions of some degenerate parabolic equations. Comm. Partial Differential Equations 18 (1993), 821-46.

17 J. E. Bouillet and C. Atkinson. A generalized diffusion equation: radial symmetries and comparison theorems. J. Math. Anal. Appl. 95 (1983), 37-68.

18 R. Dal Passo. Uniqueness of the entropy solution of a strongly degenerate parabolic equation. Comm. Partial Differential Equations 18 (1993), 265-79.

19 J. I. Diaz and F. de Thelin. On a nonlinear parabolic problem arising in some models related to turbulent flows. SIAM J. Math. Anal. 25 (1994), 1085-111.

20 J. I. Diaz and L. Veron. Local vanishing properties of solutions of elliptic and parabolic quasilinear equations. Trans. Amer. Math. Soc. 290 (1985), 787-814.

21 E. DiBenedetto. Degenerate Parabolic Equations (New York: Springer, 1993).

22 C. J. van Duijn and H. Zhang. Regularity properties of a doubly degenerate equation in hydrology. Comm. Partial Differential Equations 13 (1988), 261-319.

23 C. J. van Duyn. Some fundamental properties of the simultaneous flow of fresh and salt groundwater in horizontally extended aquifers. In Flow and Transport in Porous Media, eds A. Verruijt and F. B. J. Barends, 83-90 (Rotterdam: A. A. Balkema, 1981).

24 C. J. van Duyn and D. Hilhorst. On a doubly nonlinear diffusion equation in hydrology. Nonlinear Anal. 11 (1987), 305-33.

25 J. R. Esteban and J. L. Vazquez. On the equation of turbulent filtration in one-dimensional porous media. Nonlinear Anal. 10 (1986), 1303-25.

26 J. R. Esteban and J. L. Vazquez. Homogeneous diffusion in $\mathbb{R}$ with power-like nonlinear diffusivity. Arch. Rational Mech. Anal. 103 (1988), 39-80.

27 B. H. Gilding. The occurrence of interfaces in nonlinear diffusion-advection processes. Arch. Rational Mech. Anal. 100 (1988), 243-63.

28 B. H. Gilding. A singular nonlinear Volterra integral equation. J. Integral Equations Appl. 5 (1993), 465-502.

29 B. H. Gilding and R. Kersner. Diffusion-convection-réaction, frontières libres et une équation intégrale. C. R. Acad. Sci. Paris Sér. I Math. 313 (1991), 743-46.

30 B. H. Gilding and R. Kersner. The characterization of reaction-convection-diffusion processes by travelling waves. J. Differential Equations, 124 (1996), 27-79.

31 A. V. Ivanov. Quasilinear Degenerate and Nonuniformly Elliptic and Parabolic Equations of Second Order (Providence, Rhode Island: American Mathematical Society, 1984); originally appearing in Russian as: Trudy Mat. Inst. Steklov. 160 (1982), 5-285.

32 G. de Josselin de Jong. The simultaneous flow of fresh and salt water in aquifers of large horizontal 
extension determined by shear flow and vortex theory. In Flow and Transport in Porous Media, eds A. Verruijt and F. B. J. Barends, 75-82 (Rotterdam: A. A. Balkema, 1981).

33 J. Kačur. On a solution of degenerate elliptic-parabolic systems in Orlicz-Sobolev spaces I. Math. Z. 203 (1990), 153-71.

34 J. Kačur. On a solution of degenerate elliptic-parabolic systems in Orlicz-Sobolev spaces II. Math. Z. 203 (1990), 569-79.

35 A. D. Kalashnikov. The concept of a finite rate of propagation of a perturbation. Russian Math. Surveys 34 (1979), 235-36; originally appearing in Russian as: Uspekhi Mat. Nauk 34 (1979), 199-200.

36 A. S. Kalashnikov. A nonlinear equation arising in the theory of nonlinear filtration. Trudy Sem. Petrovsk. 4 (1978), 137-46 (in Russian).

37 A. S. Kalashnikov. On the propagation of perturbations in the processes described by degenerate parabolic equations with non-power nonlinearities. Trudy Sem. Petrovsk. 10 (1984), 118-34, 238 (in Russian).

38 A. S. Kalashnikov. Propagation of perturbations in the first boundary-value problem for a doublenonlinear degenerate parabolic equation. J. Soviet Math. 32 (1986), 315-20; originally appearing in Russian as: Trudy Sem. Petrovsk. 8 (1982), 128-34.

39 A. S. Kalashnikov. Cauchy problem for second-order degenerate parabolic equations with nonpower nonlinearities. J. Soviet Math. 33 (1986), 1014-25; originally appearing in Russian as: Trudy Sem. Petrovsk. 6 (1981), 83-96.

40 A. S. Kalashnikov. Some problems of the qualitative theory of non-linear degenerate second-order parabolic equations. Russian Math. Surveys 42 (1987), 169-222; originally appearing in Russian as: Uspekhi Mat. Nauk 42 (1987), 135-76.

41 L. S. Leibenson. Turbulent movement of gas in a porous medium. Izv. Akad. Nauk SSSR Ser. Geograf. Geofiz. 9 (1945), 3-6 (in Russian).

42 L. S. Leibenson. General problem of the movement of a compressible fluid in a porous medium. Izv. Akad. Nauk SSSR Ser. Geograf. Geofiz. 9 (1945), 7-10 (in Russian).

43 L. S. Leibenson. A fundamental law of gas motion through a porous medium. C. R. Acad. Sci. URSS 47 (1945), 16-18.

44 L. S. Leibenzon. The Motion of Natural Liquids and Gases in Porous Media (Moscow: OGIZ Gosudarstvenoe Izdatel'stva Tekhniko-Teoreticheskoi Literatury, 1947 (in Russian)).

45 J. L. Lions. Quelques Méthodes de Résolution des Problèmes aux Limites Non Linéaires (Paris: Dunod, 1969).

46 M. L. Marinov and T. V. Rangelov. Estimates on the support of the solutions for some nonlinear degenerate parabolic equations. C. R. Acad. Bulgare Sci. 39 (1986), 17-19.

47 T. Nagai. On solutions with compact support of certain nonlinear parabolic equations. Boll. Un. Mat. Ital. B 15 (1978), 9-19.

48 K. B. Pavlov (ed.). Questions of the Theory of Nonlinear Transport Processes, Trudy MVTU 374 (Moscow: Moskovskoe Vysshee Tekhnicheskoe Uchilishe, 1981 (in Russian)).

49 K. B. Pavlov. L. D. Pokrovskii and S. N. Taranenko. Properties of solutions of a nonlinear transfer equation. Differential Equations 17 (1982), 1063-8; originally appearing in Russian as: Differenstial'nye Uravneniya 17 (1981), 1661-7.

50 L. D. Pokrovskii and S. N. Taranenko. Conditions for space localization of the solutions of the nonlinear equation of heat conduction. U.S.S.R. Comput. Math. and Math. Phys. 22 (1983), 264-9; originally appearing in Russian as: Zh. Vychisl. Mat. i Mat. Fiz. 22 (1982), 747-51.

51 M. M. Porzio and V. Vespri. Holder estimates for local solutions of some doubly nonlinear degenerate parabolic equations. J. Differential Equations 103 (1993), 146-78.

52 P. A. Raviart. Sur la résolution et l'approximation de certaines équations paraboliques non linéaires dégénérées. Arch. Rational Mech. Anal. 25 (1967), 64-80.

$53 \mathrm{Yu}$. G. Rykov. The finite propagation rate of perturbations and momentum for the generalized solution of quasilinear parabolic equations. In Differential Equations and their Applications, 101-5 (Moscow: Moskovskogo Universiteta, 1984, (in Russian)).

54 Yu. G. Rykov. The behaviour with increasing time of the generalized solutions of degenerate parabolic equations with nonisotropic nonlinearities not of power type. In Differential Equations and their Applications, 160-4 (Moscow: Moskovskogo Universiteta, 1984 (in Russian)).

55 N. Su. Propagation properties for a nonlinear degenerate diffusion equation. In International Symposium on Nonlinear Problems in Engineering and Science, eds S. Xiao and X.-C. Hu, 137-47 (Beijing: Science Press, 1992).

56 N. Su. On propagation properties of perturbation in nonlinear degenerate diffusions with convection and absorption. Nonlinear Anal. 23 (1994), 1321-30.

57 M. Tsutsumi. On solutions of some doubly nonlinear degenerate parabolic equations with absorption. J. Math. Anal. Appl. 132 (1988), 187-212. 
58 V. Vespri. Analytic semigroups, degenerate elliptic operators and applications to nonlinear Cauchy problems. Ann. Mat. Pura Appl. (4) 155 (1989), 353-88.

59 V. Vespri. Abstract quasilinear parabolic equations with variable domains. Differential Integral Equations 4 (1991), 1041-72.

$60 \mathrm{~V}$. Vespri. On the local behaviour of solutions of certain singular parabolic equations. Nonlinear Anal. 16 (1991), 827-46.

61 V. Vespri. On the local behaviour of solutions of a certain class of doubly nonlinear parabolic equations. Manuscripta Math. 75 (1992), 65-80.

$62 \mathrm{~J}$. Yin. On a class of quasilinear parabolic equations of second order with double-degeneracy. J. Partial Differential Equations 4 (1990), 49-64.

63 J. Yin. Solutions with compact support for nonlinear diffusion equations. Nonlinear Anal. 19 (1992), 309-21.

64 J. Yin. Parabolic equations with both non-convexity nonlinearities and strong degeneracy. Acta Math. Sci. (English Edn.), 14 (1994), 451-60.

(Issued 19 August 1996) 